Journal OF OBJECT TECHNOLOGY

Published by AITO - Association Internationale pour les Technologies Objets

http://www.jot.fm/

\title{
Architecture Conformance Checking in Dynamically Typed Languages
}

\author{
Sergio Miranda ${ }^{\mathrm{a}} \quad$ Elder Rodrigues Jr ${ }^{\mathrm{b}} \quad$ Marco Tulio Valente ${ }^{\mathrm{a}}$ \\ Ricardo Terra ${ }^{\mathrm{b}}$ \\ a. Department of Computer Science, Federal University of Minas Gerais \\ b. Department of Computer Science, Federal University of Lavras
}

\begin{abstract}
Architectural erosion is a recurrent problem faced by software architects, which might be even more severe in systems implemented in dynamically typed languages. The reasons are twofold: (i) some features provided by such languages make developers more propitious to break the planned architecture (e.g., dynamic invocations and buildings), and (ii) the developers' community lacks tool support for monitoring the implemented architecture. To address these shortcomings, this paper presents an architectural conformance and visualization approach based on static code analysis techniques and on a lightweight type propagation heuristic. The central idea is to provide the developers' community with means to control the architectural erosion process by reporting architectural violations and visualizing them in high-level architectural models, such as reflexion models and DSMs. This paper also describes a tool - called ArchRuby - that implements our approach. We evaluate our solution in three real-world systems identifying 48 architectural violations of which the developers had no prior knowledge. We also measure the effectiveness of our type propagation heuristic reporting that (i) the number of analyzed types raises $5 \%$ on the average and (ii) certain violations are only detected due to our heuristic.
\end{abstract}

Keywords Architecture conformance checking; high-level architectural models; dynamically typed languages.

\section{Introduction}

The planned architecture of a system comprises a set of standards and best practices that enable its evolution [Par94]. However, as the project evolves - due to lack of knowledge, short deadlines, etc.- - these patterns tend to deteriorate and hence nullifying the benefits provided by an architectural design, such as maintainability, scalability, portability, etc. [PTD ${ }^{+} 10$, MNS95]. This phenomenon is known as software

Sergio Miranda, Elder Rodrigues Jr, Marco Tulio Valente, Ricardo Terra. Architecture Conformance Checking in Dynamically Typed Languages. Licensed under Attribution-NoDerivatives 4.0 International (CC BY-ND 4.0). In Journal of Object Technology, vol. 15, no. 3, 2016, pages 1:1-35. doi:10.5381/jot.2016.15.3.a1 
architecture erosion and it is considered a challenging research problem in the software architecture area [KMHM08, TVCB15, $\mathrm{SRK}^{+}$09, Bor11, Bos04]. This erosion process might be even more severe in systems implemented in dynamic languages for two main reasons: (i) some features provided by these languages (e.g., dynamic invocations, dynamic buildings, eval, etc.) make the developers more likely to break the planned architecture, and (ii) these languages suffer from the lack of architecture and design tools.

This article is centered on the assumption that systems implemented in dynamic languages should also benefit from architectural conformance techniques and tools. More important, existing architectural conformance solutions are limited since, as far as we know, none addresses the particularities of dynamically typed languages. Therefore, in a previous conference paper [MVT15b], we were the precursors in proposing an architectural conformance checking approach for systems implemented in dynamic languages, which is based on static code analysis techniques and on a lightweight type propagation heuristic. Although our focus was on the architecture conformance checking, we also provided high-level architectural models to better visualize the identified violations. Furthermore, we showed that it is possible to monitor the architecture of these systems using our proposed approach, which is non-invasive and hence does not modify the source code or impact on the performance. As a practical contribution, we implemented a tool for Ruby that supports a simple and objective way to detect architectural violations and to visualize them using two high-level architectural models, namely Reflexion Models and Dependency Structure Matrices (DSMs).

In the presented paper, we extend our work in the following directions: (a) by including more details and one more system in the evaluation of the proposed approach. As a result, we could detect 48 violations in three real-world systems of which the developers had no prior knowledge; (b) by evaluating the effectiveness of our lightweight type propagation heuristic in the three previously evaluated real-world systems, besides complementarily in 28 open-source systems. As main findings, the number of inspected types increases $5 \%$ on the average but up to $17 \%$ with our heuristic, and some violations are only detected due to this heuristic; (c) by applying ArchRuby in itself we illustrate our approach; and (d) by providing more technical details on the ArchRuby implementation.

The remainder of this paper is organized as follows. Section 2 provides a definition for central concepts needed to follow our approach, such as Ruby features, architecture conformance checking, and high-level architectural models. Section 3 presents the proposed approach, describing the architectural rules specification, conformance, and visualization processes. Section 4 details the proposed type propagation heuristic. Section 5 presents ArchRuby, the tool that implements the proposed solution. Section 6 reports results from applying our solution in three real-world systems. Section 7 measures the effectiveness of the proposed type propagation heuristic in the previous evaluated systems. Finally, Section 8 discusses related work and Section 9 concludes.

\section{Background}

In this section, we discuss background related to our work. Section 2.1 describes and exemplifies Ruby features, and Section 2.2 briefly introduces architectural conformance checking techniques and high-level architectural models. 


\subsection{Ruby}

Since our approach focuses on systems implemented in Ruby, an overview of the language features is relevant. Every Ruby program is designed through objects, since the language is purely objected-oriented [Bla09]. Even true and false are objects, i.e., they are instances of TrueClass and FalseClass, respectively. To define classes and methods, we use the class and def keywords, respectively. Ruby has single inheritance, but it is possible to include many modules in one class. Basically, a module is used to implement methods and constants but, unlike a class, it is not possible to instantiate a module.

Furthermore, Ruby is a dynamic language with several powerful abstractions. For example, it is possible to define methods, classes, re-open a class, evaluate a valid Ruby code inside some context, re-define methods and call methods passing strings as arguments. As an example, Figure 1 illustrates the usage of the aforementioned features. In line 1 , the code is defining a class RbClass and a module Test. In line 2, class RbClass includes module Test, therefore method salute is now part of this class. In line 7 we instantiate an object of RbClass and call method say hi (line 8). In line 10, we re-open class RbClass and define a new method called say_bye (lines 11-13). In lines 16-18, we define a method add only for the object o. Finally, in line 20, we call the method defined by last.

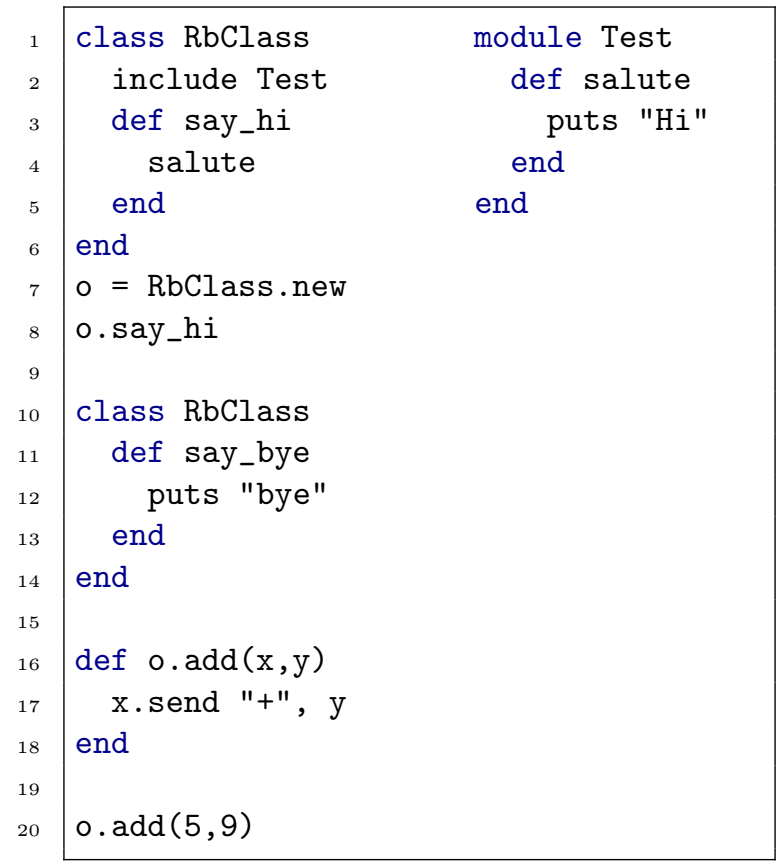

Figure 1 - Ruby source code example

\subsection{Architectural Conformance and Visualization}

Architecture is a crucial artifact that needs to be followed and monitored by the developers during software development. Architectural conformance is the process that checks to which degree the concrete architecture (e.g., the source code implementation) is consistent with the planned one [KMR08]. Architectural conformance can be static 
(i.e., without executing the target system) or dynamic (i.e., executing the target system). This section presents architecture conformance checking techniques related to ArchRuby:

Reflexion Models (RMs): As proposed by Murphy et al., Reflexion Models compare two models [MNS95]. One representing a high-level model of the system (e.g., specified by the developer) and another representing the low-level model of the system (e.g., produced either by statically analyzing the system source or by collecting information during the system execution). With these inputs the technique computes a software reflexion model that matches the high-level and the low-level models. The reflexion model highlights divergences and absences, regarding these models. Divergences indicate source interactions that are not expected by the planned architecture and absences indicate interactions that are expected but not found. The outcome of the evaluation is usually summarized and documented in a separated report, which is presented as a graph and text. The former connects system modules and reports the detected divergences and absences.

Dependency Structure Matrices (DSMs): The concept of DSM was first proposed by Baldwin and Clark to show the importance of modular design in the hardware industry [BC99]. Thereafter, Sullivan et al. claimed that DSM could also be used in software industry [SGCH01]. A DSM is a square matrix where the rows and columns represent the modules of the system. Traditionally, DSM used a "X" to indicate a dependency between two modules. However, Sangal et al. in the LDM tool represent in the cells the number of references between two modules [SJSJ05]. In this tool, it is possible to distinguish the dependencies using design rules, which have two forms: $A$ can-use $B$ and $A$ cannot-use $B$, indicating that module $A$ can (or cannot) depend on module $B$. DSM has a more scalable output than the output generated by reflexion models based on graphs, since a matrix usually scales better than a graph.

Constraint languages: The main objective of constraint languages is to provide a method to specify structural dependencies. DCL (Dependency Constraint Language) is a domain specific language that supports the definition of structural constraints between modules [TV09]. DCL provides constraints to capture divergences and absences. First, to capture divergence architects have to specify only can, can only or cannot rules for specified modules. Last, to capture absence architects specify dependencies that must be present in the source code. ArchRuby - the architecture conformance checking technique proposed in this paper - is directly inspired on DCL constraints.

\section{The Proposed Approach}

This paper describes an architectural conformance approach based on static code analysis techniques and on a lightweight type propagation heuristic for systems implemented in dynamically typed languages. Additionally, we also provide two highlevel architectural models to better visualize the architectural violations. The central goal is to provide developers with means to control the architectural erosion process by reporting architectural violations (conformance) and by providing the high-level architectural models to better visualize the identified violations (visualization). 
Figure 2 provides an overview on the proposed approach. Our solution receives as input the architectural rules (in1) and the source code of the target system (in2). After parsing the architectural rules file ( $t 1)$ and the source code ( $t 2)$, it triggers the architectural conformance process (t3) in order to detect design decisions that do not respect the intended architecture. As result, our solution outputs a textual report (out1), which details the detected violations (source code location, violated rule, etc.), and two high-level architectural models to better visualize the identified violations (out2). In these models, we differentiate the dependencies - edges in reflexion models and cells in DSMs - that represent violations (refer to Section 2.2).

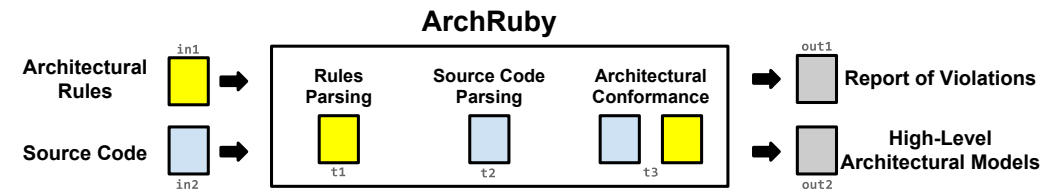

Figure 2 - The proposed approach

This section is organized as follows. Section 3.1 presents the running system. Section 3.2 details the specification of architectural rules and Section 3.3 describes the architectural conformance process. Finally, Section 3.4 describes the high-level architectural models our approach relies on to better visualize the identified violations.

\subsection{Running Example}

We rely on the architecture of ArchRuby ${ }^{1}$ itself and its implementation to illustrate the architectural conformance and visualization processes provided by our approach. The tool was implemented in Ruby and relies on five Gems: ${ }^{2}$ RubyParser to parse the source code, SexpProcessor to perform tree traversals, Yaml to parse the architectural rules specification file, GraphViz to produce the reflexion model, and IMGKit to produce the DSM. Figure 3 shows the diagram of the core classes of the system. A more detailed description on the ArchRuby implementation can be found in Section 5.

\subsection{Architectural Rules Specification}

Architectural rules are specified in a domain-specific language in YAML format, widely used in the Ruby ecosystem. Thereupon, even non-experienced developers can easily define rules. Specifically, each module of the system under evaluation must be formalized as follows: ${ }^{3}$

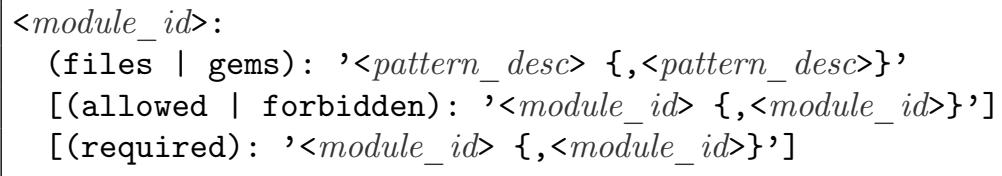

where $<$ module_id $>$ is the name of the module (line 1). Modules can be composed by files (files) or Gems (gems) that must be defined by at least one <pattern_desc $>$,

\footnotetext{
${ }^{1}$ The source code is publicly available at http://github.com/sergiotp/archruby.

${ }^{2} \mathrm{Gem}$ represents a reusable package or application written in Ruby language.

${ }^{3}$ Formalization based on the Extended Backus-Naur Form (EBNF).
} 


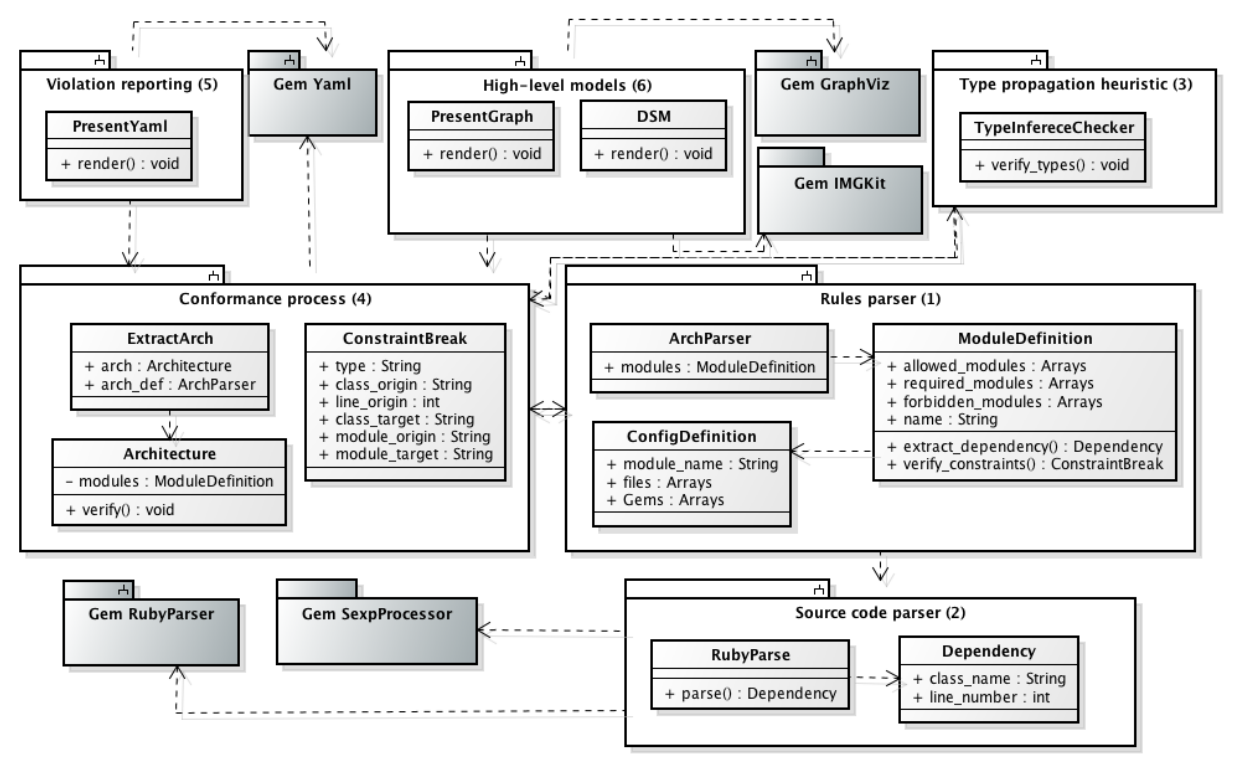

Figure 3 - ArchRuby architecture

delimited by commas (line 2). It is not possible to (i) combine files and Gems in the same module definition, and (ii) define constraints to module composed strictly by Gems, since they are external libraries that are not part of the target system being analyzed. When specifying files, the pattern matching is based on shell glob ${ }^{4}$ (a default Ruby file library) to map multiple files at once using wildcards, e.g., $*$ and $* *$.

To detect divergences - dependencies that exist in the source code but are not prescribed by the planned architecture $\left[\mathrm{PTD}^{+} 10\right]$ - for each module we define the ones that it is allowed to depend (allowed) or not (forbidden), which are defined by at least one $<$ module_id $>$, delimited by commas (line 3 ). Here, we consider as a dependency from a type $A$ to a type $B$ when (i) $A$ accesses a field of type $B$ (ii) $A$ invokes a method of type $B$, (iii) $A$ instantiates an object of type $B$, (iv) $A$ declares a variable or formal parameter of type $B,(\mathrm{v}) A$ raises an exception of type $B$, and (vi) $A$ inherits from, extends, or includes $B .{ }^{5}$ Likewise, to detect absencesdependencies that do not exist in the source code but are required by the planned architecture $\left[\mathrm{PTD}^{+} 10\right]$ - for each module we define the ones that it must depend (required), which are defined as aforementioned (line 4). It is worth noting that a definition for a particular module can combine required with allowed or forbidden. However, it cannot have allowed and forbidden in a same module definition. When a module does not define clauses allowed and forbidden, our language considers that such module is allowed to depend on any module.

In order to illustrate an YAML definition, Figure 4 presents the definition of

\footnotetext{
${ }^{4} \mathrm{~A}$ detailed explanation of shell glob in Ruby (specifically, class Dir) can be found at: http : //ruby-doc.org/core-2.2.0/Dir.html\#method-c-glob

${ }^{5}$ The code of a lambda is verified only in the method where it is defined, not in its call sites. For instance, assume that a method return_lambda in module $M_{3}$ returns a lambda $f$. Assume also that a module $M_{2}$ defines a method search lambda that calls $M_{3}$ ::return lambda. Assume, lastly, that a method in module $M_{1}$ calls $M_{2}:$ :search_lambda. In such scenario, (i) only module $M 3$ depends on the types lambda $f$ establishes dependency with, (ii) module $M_{1}$ depends only on module $M_{2}$ and (iii) module $M_{2}$ depends only on module $M_{3}$.
} 
architectural rules to the ArchRuby tool. For example, module module_definition (lines 1-3) contains file module definition.rb and can depend on classes from module config_definition, ruby_parser, dependency, constraint_break, and file_extractor. On the other hand, module multiple_constraints_validator (lines 5-7) contains file archruby.rb and cannot depend on classes from module architecture. Moreover, shell glob allows to use $*$ to reference all files in the directory and $* *$ to reference directories in a recursive manner. For example, module presenters (line 13) is composed by all $\mathrm{rb}$ files listed in directories inside presenters. It is worth noting that we do not define architectural rules for modules strictly composed by Gems (e.g., parser_ruby, sexp_processor, yaml_parser, and graphviz) because they are not internal components of the target system. Nevertheless, Gems must be defined by their namespace (main module). For example, module parser_ruby is composed by Gem ruby_parser whose namespace is RubyParser (lines 41-42).

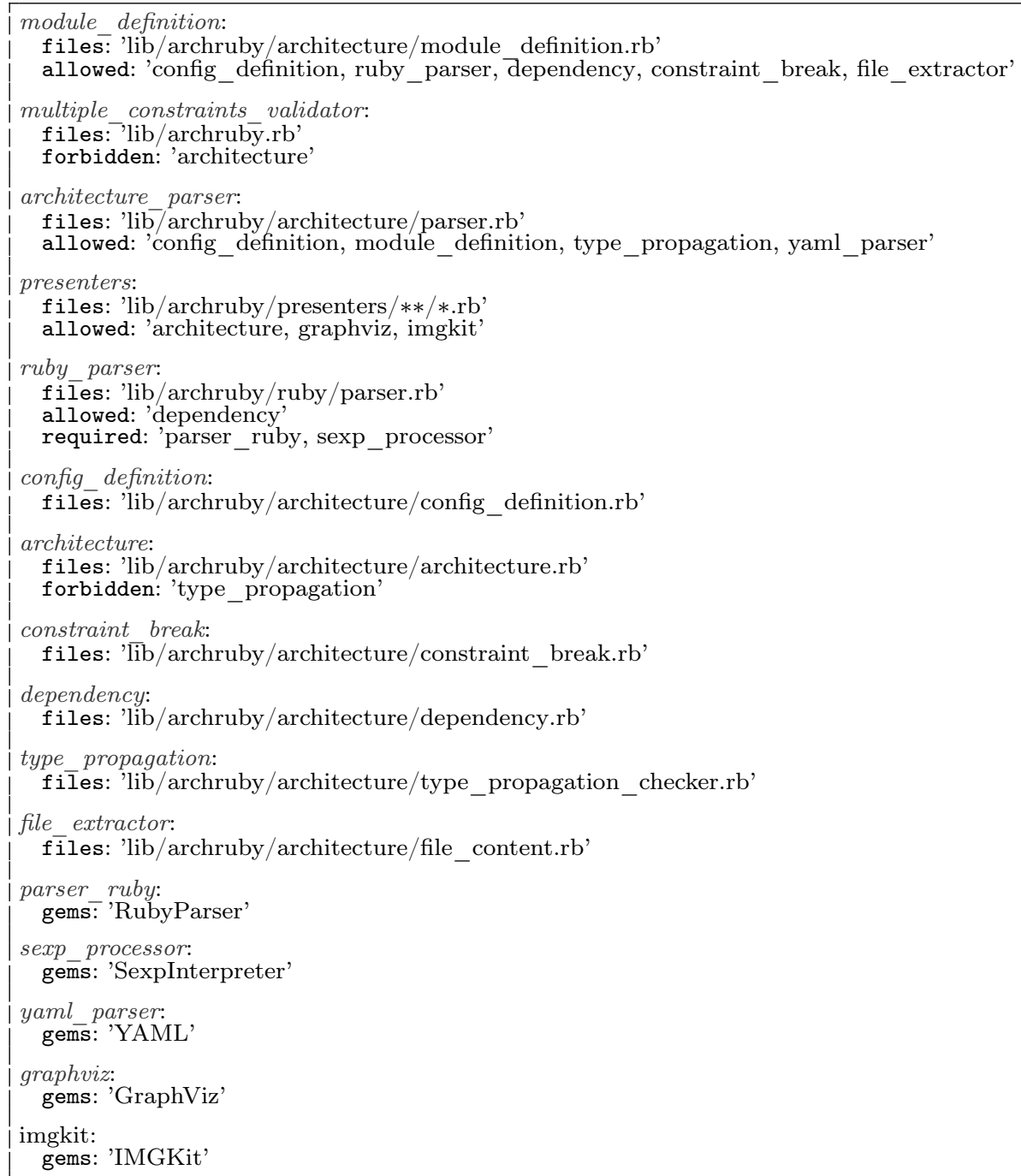

Figure 4 - ArchRuby architectural specification file 


\subsection{Architectural Conformance}

The architectural conformance process is performed from the architectural rules specification and the source code of the target system. This process (i) extracts the modules and rules from the architectural rules specification file; (ii) extracts the dependency graph of the entire system; (iii) includes type information in the dependency graph using a type propagation heuristic (described in Section 4); and (iv) checks whether the dependencies obtained in steps $i i$ and $i i i$ respect the rules defined in step $i$.

The conformance process outputs a file reporting the detected architectural violations (divergences and absences). For example, consider the rules defined for ArchRuby (Figure 4). In such specification, module module_definition is not explicitly allowed to depend on module type_propagation (line 3 ). However, assume that a class from module_definition accesses a class from type_propagation. Such dependency represents a violation and would be reported to developers in the textual output file as illustrated in Figure 5. ${ }^{6}$ For each detected violation, the report indicates the violation type (line 1), information from the origin class (lines 2-4) and from the target class (lines 5-6), and the violated rule (line 7). Besides the textual report file, ArchRuby also provides two graphical report files in order to provide complementary ways to visualize the detected violations, as explained in Section 3.4.

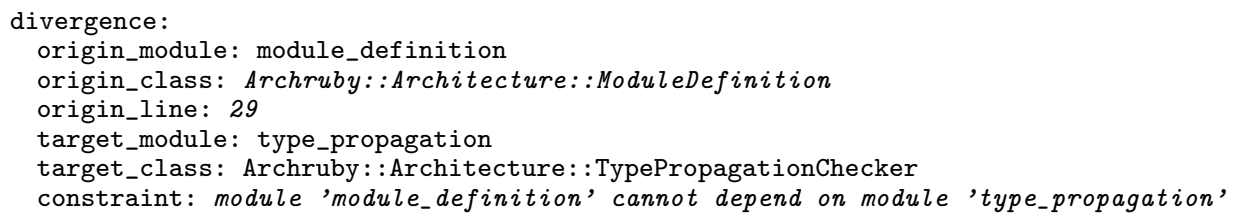

Figure 5 - Textual report of an architectural violation

\subsection{Architectural Visualization}

Although we focus on architecture conformance checking process, we complement our textual report of violations by providing two high-level architectural models to better visualize the identified violations: (i) Reflexion Model in a subtle adaptation of the one originally proposed by Murphy et al. [MNS95] and (ii) Dependency Structure Matrix (DSM) in a subtle adaptation of the one proposed by Sangal et al. [SJSJ05].

\subsubsection{Reflexion Model}

The reflexion model is a directed dependency graph whose vertices represent the modules defined in the architectural rules specification and edges represent dependencies established between the modules, which are differentiated when refer to architectural violations (refer to Section 2.2).

Figure 6 illustrates the reflexion model of ArchRuby. ${ }^{7}$ The light gray rectangles represent internal modules (e.g., module definition) and the gray trapezes represent external modules (e.g., parser_ruby). The edges are shown as follows (assume an edge from $A$ to $B)$ :

\footnotetext{
${ }^{6}$ The report is also in YAML format to facilitate reuse.

${ }^{7}$ For a better visualization, all high-level architectural models - Reflexion Models and DSMs-are publicly available at: http : //aserg.labsoft.dcc.ufmg.br/archruby/jot2016
} 


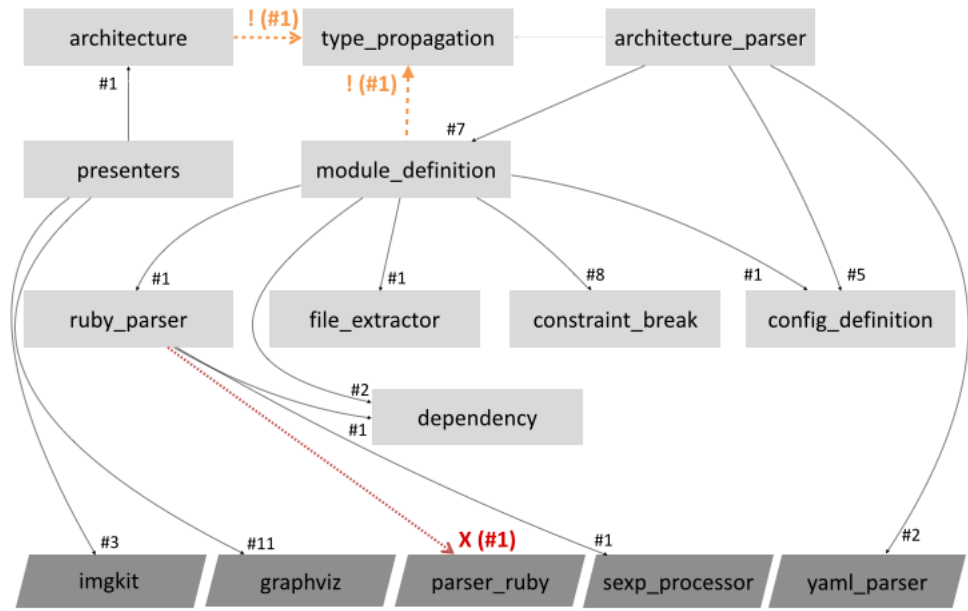

Figure 6 - Reflexion model automatically computed by ArchRuby

$(\rightarrow)$ Black edge: indicates an allowed dependency from module $A$ to $B$. For instance, ruby_parser establishes one (\#1) dependency with module dependency (see line 19, Figure 4).

$(\stackrel{b}{)}$ Dashed orange edge with an "!" mark: indicates a divergence, i.e., there is a class from module $A$ depending on module $B$, even though it is (i) forbidden or (ii) not explicitly allowed. For example, architecture depends on module type propagation, but it is forbidden (case $i$; see line 27, Figure 4). As another example, module_definition depends on module type_propagation, but it is not explicitly allowed (case $i i$; see line 3, Figure 4).

$(\stackrel{x}{*})$ Dotted red edge with an "X" mark: indicates an absence, i.e., there is no class from module $A$ depending on module $B$, even though it is required. For instance, a class from ruby_parser does not depend on parser_ruby (see line 20, Figure 4).

$(\rightarrow)$ Gray edge: indicates a warning, i.e., there is no class from module $A$ depending on module $B$, even though it is prescribed as allowed. For instance, we defined that architecture_parser is allowed to depend on module type_propagation (see line 11, Figure 4), but there is no dependency from the former to the latter.

\subsubsection{Dependency Structure Matrix}

Reflexion models have a well-known scalability problem since it is a graph-based model. As the number of modules and dependencies grows, the model becomes unreadable. In this sense, ArchRuby also provides a high-level architectural model based on DSMs, 
which is a weighted square matrix where the rows and columns are numbered and represent the modules of the system, and the cells represent the dependencies between them (refer to Section 2.2).

Figure 7 illustrates the DSM of ArchRuby. The cells represent the number of references between two modules. The cells are shown as follows:

( ) Gray cell: indicates a allowed dependency. For instance, the number 7 in row 1 and column 3 denotes that module architecture_parser establishes seven allowed dependencies with module module_definition.

( ) Orange cell: indicates a divergence. For example, the number 1 in row 10 and column 7 represents that module architecture establishes a forbidden dependency with module parser ruby. As another example, the number 1 in row 10 and column 1 represents that module module definition establishes a forbidden dependency with module type_propagation.

( column 5 represents that module ruby_parser does not depend on module parser_ruby even though it is required.

2 ) Question cell: indicates a warning. For instance, the symbol "?" in row 10 and column 3 represents that module architecture_parser does not establish an expected dependency with module type_propagation.

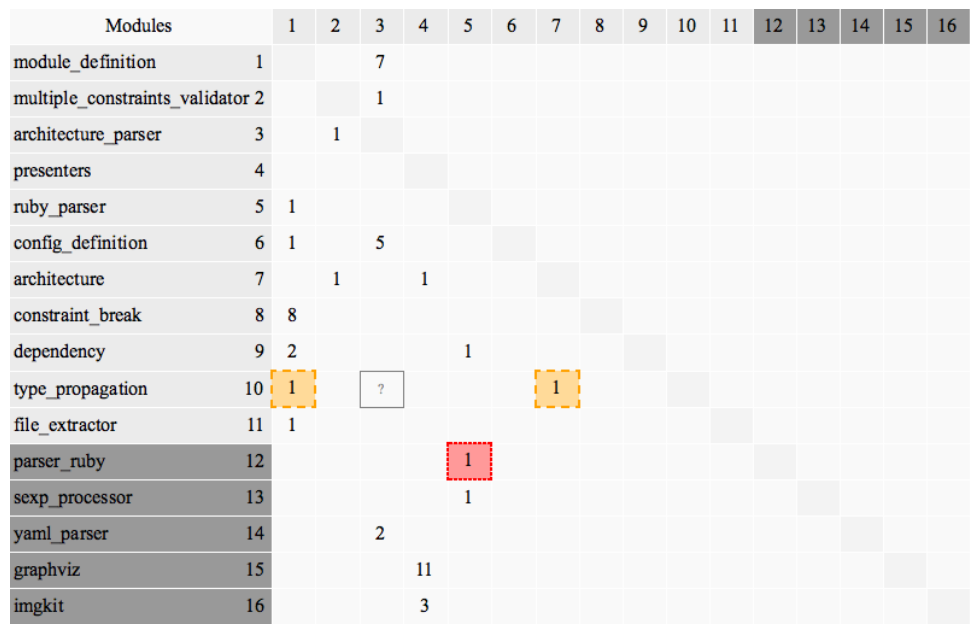

Figure 7 - DSM automatically computed by ArchRuby

\section{The Proposed Type Propagation Heuristic}

In this section, we describe a type propagation heuristic - more specifically, a simplification of the one formalized by Furr et al. [FhDAFH09] — which aims to build a set TYPES whose elements are triples [method, var name, type], where type is one of the possible types inferred for a variable or a formal parameter var name defined in method method. We build this set based on the following recursive definition: 
i) Base: For each direct inference (e.g., instantiation) of a type $\mathrm{T}$ assigned to a variable $\mathrm{x}$ in a method $\mathrm{f}$, then $[\mathrm{f}, \mathrm{x}, \mathrm{T}] \in$ TYPES.

ii) Recursive step: If $[f, x, T] \in$ TYPES and there is a call $g(x)$ in $f$, then $[g, y, T] \in$ TYPES, where $\mathrm{y}$ is the name of the formal parameter in $\mathrm{g}$. This step is applied until a fixpoint is reached, i.e., no new triples are added to set TYPES.

Figure 8 illustrates the proposed heuristic. When executing the base step of the algorithm, it initializes TYPES with $[A:: f, x, F o o],[A: f, b, B],[A:: f$, self, A], [B::g, c, C], and $[C:: h, d, D]$ since they can be directly inferred. On the first application of the recursive step, the triples $[\mathrm{B}: \mathrm{g}, \mathrm{x}$, Foo] and $[\mathrm{B}:: \mathrm{g}, \mathrm{z}, \mathrm{A}]$ are included in TYPES, since the type of the variables $x$ and self are known in the call of $g$. On the second application of the recursive step, the triples [C::h, y, Foo] and [C::h, y, A] are included in TYPES, since the type of variables $\mathrm{x}$ and $\mathrm{z}$ are known in the call of $\mathrm{h}$. On the third application of the recursive step, the triples [D::m, $\mathrm{k}$, Foo] and [D::m, $\mathrm{k}, \mathrm{A}]$ are included in TYPES (where $\mathrm{k}$ is the name of the parameter in $\mathrm{D}: \mathrm{m}$ ), since the type of the variable $\mathrm{y}$ is known in the call of $\mathrm{m}$. The forth application of the recursive step reaches the fixpoint since no new triple is added to set TYPES.

\begin{tabular}{|ccc|}
\hline class A & class B & class C \\
def $f$ & def $g(x$ z $)$ & def $h(y)$ \\
$x=$ Foo.new & $c=C . n e w$ & d = D.new \\
b = B.new & $c \cdot h(x)$ & d.m(y) \\
b.g(x,self $)$ & $c \cdot h(z)$ & end \\
end & end & end \\
end & end & \\
\hline
\end{tabular}

Figure 8 - Piece of code to illustrate the proposed type propagation heuristic

In this example, it is worth noting that the formal parameter y of method $\mathrm{C}: \mathrm{h}$ can be either of type A or Foo. It indicates that: (i) the type propagation mechanism has to consider all potential types of a variable or formal parameter when propagating the type; and (ii) the architectural conformance process has also to consider all potential types (A and Foo, in this scenario) when searching for violations.

\section{The ArchRuby Tool}

ArchRuby is a Gem for Ruby that implements our proposed approach [MVT15a]. The tool is executed from the command line. We decided for such UI because, in such way, any organization - regardless of its software environment - can adopt ArchRuby in its development process. The following example illustrates a usage scenario:

archruby --arch_def_file=/fmot/arch_def.yml --app_root_path=/fmot

The executable archruby requires as input the path of the architectural rules file (--arch_def_file) and the path of the system (--app_root_path), and provides as output the architecture violation report (archruby_report.yml) and two high-level 
architectural models to better visualize the identified violations (archruby rm.png and archruby_dsm.png), as previously illustrated in Figure 2.

As also previously illustrated in Figure 3, the ArchRuby implementation follows an architecture divided in the following modules:

1. Rules parser: Responsible for extracting and storing the content of the architectural rules file (e.g., /fmot/arch_def.yml) in an internal data structure. It also warns the user when he/she specifies invalid constraints, e.g., allowed and forbidden together. We rely on the standard Ruby Yaml Gem to parse the YAML file.

2. Source code parser: Responsible for extracting and storing all system dependencies (e.g., from /fmot) in an internal data structure. We rely on Gem ruby_parser to parse the source code of each class. It produces $s$-expressions, which are data structures in form of tree. Basically, during the tree traversal, this module stores the type of variables and formal parameters, besides the calls involving them.

3. Type propagation heuristic: Responsible for inferring types of variables, according to the heuristic previously described in Section 4. It complements the internal data structure obtained by the Source code parser module.

4. Conformance process: Responsible for verifying whether the implemented architecture (as represented by the source code) follows the planned architecture (as represented by the architectural rules), as previously described in Section 3.3. This module detects the dependencies that do not respect the specified architectural rules and stores detailed information regarding them. It relies on the data structures initially built by the Rules parser and Source code parser modules to detect the dependencies that do not respect the architectural rules. In other words, this module analyzes the internal data structure built in the previous steps to search for potential violations. When a violation is detected, it stores detailed information-namely dependency type, name of the source and target modules, line number, and name of the source and target classes (see Figure 3, class ConstraintBreak) — for further reference.

5. Violation reporting: Responsible for structuring the detected architectural violations in a YAML file (archruby_report.yml).

6. High-level models: Responsible for generating the high-level architectural models of the target system as previously described in Section 3.4. It relies on the data structure initially built by the Source code parser module and on the set of violations detected in the Conformance process module to highlight the identified violations in the generated visualization models. This module relies on Gem GraphViz to produce reflexion models as annotated directed dependency graphs and on Gem IMGKit to produce DSMs as HTML tables with CSS style.

Although each of the aforementioned modules has a well-defined responsibility, they may contain more than one single class in order to have a greater control over the parts of the system. In such way, it is easier to maintain the existing features and add new ones. Furthermore, we have implemented several unit tests that are automatically 
performed during regression testing to ensure that changes do not break the expected behavior of the system. It is worth noting that the dependencies that are not part of the Ruby standard library (e.g., ruby_parser and GraphViz) are automatically installed when the user installs ArchRuby.

\section{Evaluation of the Proposed Approach}

This section evaluates the applicability of our proposed approach in real contexts of software development. We chose three real-world systems - Dito Social, Tim Beta, and PLC Attorneys - to apply our architecture conformance checking process. For each system, we report the results into each step: (i) architectural rules specification, (ii) architectural conformance, and (iii) architectural visualization. More important, a qualitative discussion is conducted for each evaluated system, besides a general discussion to conclude the section.

\subsection{Target Systems}

We evaluate our solution in three real-world systems: ${ }^{8}$ Dito Social, a social platform provided by an IT company to its final customers; Tim Beta, a telecommunication company communication channel with mostly target young groups; and PLC Attorneys, a project task management software system used by a law firm. Table 1 reports the main information of the systems.

Table 1 - Target systems

\begin{tabular}{lccl}
\hline System & LOC & $\begin{array}{c}\text { \# classes } \\
\text { \# gems }\end{array}$ & Technologies \\
\hline Dito Social & 13,304 & $142 / 34$ & $\begin{array}{l}\text { Ruby on Rails, Resque, Rspec, RSA, Twitter, } \\
\text { Google Plus, Koala, Suspot Rails, Mysql2 }\end{array}$ \\
Tim Beta & 17,817 & $141 / 50$ & $\begin{array}{l}\text { Ruby On Rails, Resque, Twitter, YoutubeIt, } \\
\text { Google Plus, Instagram, Devise, Foursquare2 }\end{array}$ \\
PLC Attorneys & 2,034 & $52 / 35$ & $\begin{array}{l}\text { Ruby on Rails, Devise, CanCanCan, PaperClip, } \\
\text { Mysql2, Select2Rails, CoffeeRails }\end{array}$ \\
\hline
\end{tabular}

\subsection{Methodology}

For each subject system with the support of its chief architect who designed the evaluated architecture, we performed the following major steps:

(i) Architectural rules specification: The software architect defines the planned architecture of the system, soon after be instructed on how to specify modules and rules using our architectural description language (Section 3.2). To ensure the correct understanding by the architects, we ask them to practice the specification in an illustrative project. During the practice, they must specify a few rules and they can ask for clarifications. By concluding the practice, we argue that the architects are fully qualified to specify the architectural rules.

(ii) Architectural conformance: After a brief tutorial about our tool-its inputs and outputs - the software architect executes ArchRuby and validates the detected violations. Occasionally, the software architect can refine the architectural

\footnotetext{
${ }^{8}$ http://www.dito.com.br, http://www.timbeta.com.br, and http://metodo.plcadvogados.com.br
} 
rules - which have been specified in step (i) - to avoid false positives. Specifically, we ask the architects to analyze each violation and double check in the source code whether the violation is indeed a true positive. We repeat this process until the architects are confident that the architectural rules indeed represent the system architecture.

(iii) Architectural visualization: The software architect evaluates the reflexion model, one of the high-level architectural models provided by ArchRuby, to better visualize the identified violations. We ask the architects to express an opinion on the readability and representativeness of the reflexion model. Occasionally, to provide a more solid feedback, the architects can share the model with other team members.

\subsection{Dito Social}

Architectural rules specification: The software architect specified 62 modules and 43 architectural rules. A relevant subset of the specification is reported in Figure 9. ${ }^{9}$ The dashboard_controller module is responsible for presenting information to the customers and hence can access several data providers' modules (lines 3-7). The facebook_info_retriever module is responsible for retrieving data from Facebook and hence can access only the modules facebook and airbrake (line 11). The post model is responsible for data persistence and hence must implement classes from module activerecord (line 15) and can access modules that provide underlying services (lines 16-18), e.g., post_workers. The report_model module is responsible for generating reports about posts and interactions, and hence can access modules that provides data and e-mail delivery functionality (e.g., post_model, interaction_model, mail, aws, etc.) (line 22).

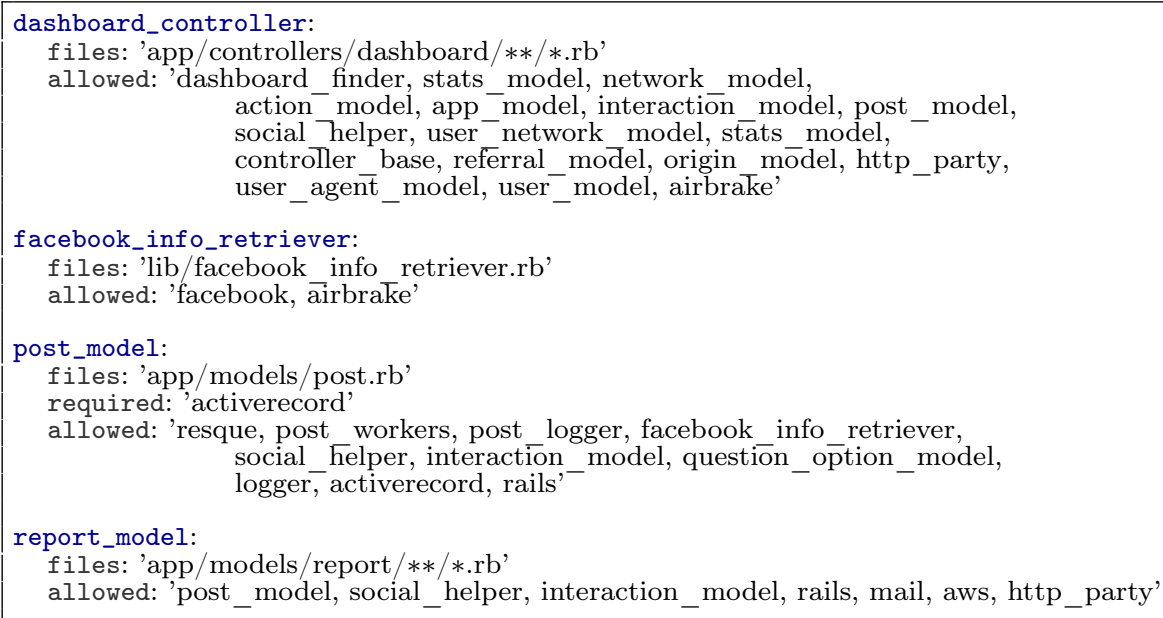

Figure 9 - Subset of the architectural specification of Dito Social

Architectural conformance: ArchRuby could detect 24 violations in Dito Social, as reported in Table 2. Two of these violations are discussed next.

\footnotetext{
${ }^{9}$ The complete data of the evaluation of the proprietary systems are available at: http : //aserg.labsoft.dcc.ufmg.br/archruby/jot2016
} 
Table 2 - Architectural violations detected in Dito Social

\begin{tabular}{lllc}
\hline Module & Rules & & \# Violations \\
\hline dashboard_controller & allowed: 'dashboard_finder, ...' & 16 \\
dashboard_finder & allowed: 'stats_model, ..., & 3 \\
report_model & allowed: 'post_model, ...' & 2 \\
event_model & allowed: 'action_model, & 1 \\
user_model & allowed: 'user_infos, ...' & 1 \\
facebook_info_retriever & allowed: 'facebook, airbrake' & 1 \\
\hline
\end{tabular}

Example of violation \#1: The service of user notification (e.g., e-mail) was moved to another system and hence it is no longer part of Dito Social. Nonetheless, as shown in Figure 10, ArchRuby detected five dependencies (lines 2, 3, 5, and 7) in class EmailsController - which belongs to module dashboard_controllerto class Email, which is not explicitly allowed according to the architectural rules (lines 3-7 of Figure 9). More specifically, class Email does not belong to any defined module; in this case, we include such kind of classes in a module called unknown.

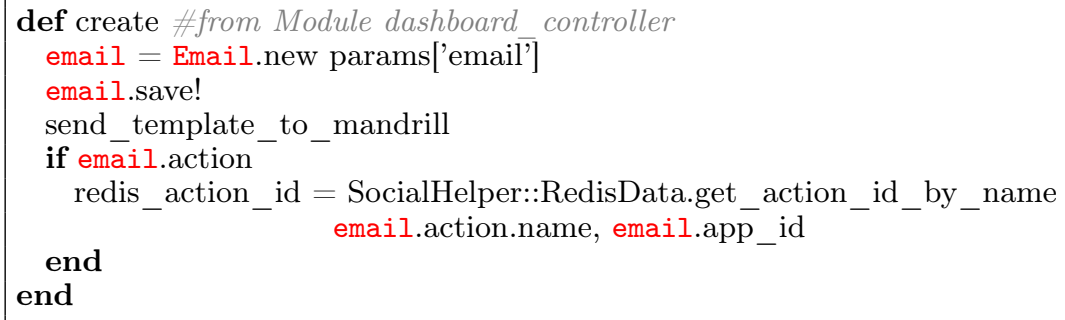

Figure 10 - Example \#1 - Divergence detected in Dito Social

Example of violation \#2: Module post_model is allowed to access module facebook_info_retriever, but not the opposite. Nevertheless, as shown in Figure 11, ArchRuby detected two dependencies (lines 15 and 18) in class FacebookInfoRetriever - which belongs to module facebook_info_retriever - to class Post from module post_model, which is not allowed according to the architectural rules (line 11 of Figure 9). It is worth noting that our approach could only detect such violation due to our type propagation heuristic, since the type was first inferred in class Post (line 5), but it was propagated by the method call to get_first_likes_comments_and_people (lines 8-9).

Architectural visualization: Figure 12 illustrates a fragment of the reflexion model. We can note divergences (orange edges) from modules dashboard_controller (as described in Example \#1), event_model, user_model, report_model, and dashboard_finder to classes that do not belong to any defined module. We also can note the allowed communication from module post model to facebook_info_retriever (black edge). However, the opposite, as described in Example $\# 2$, is highlighted as a divergence. 


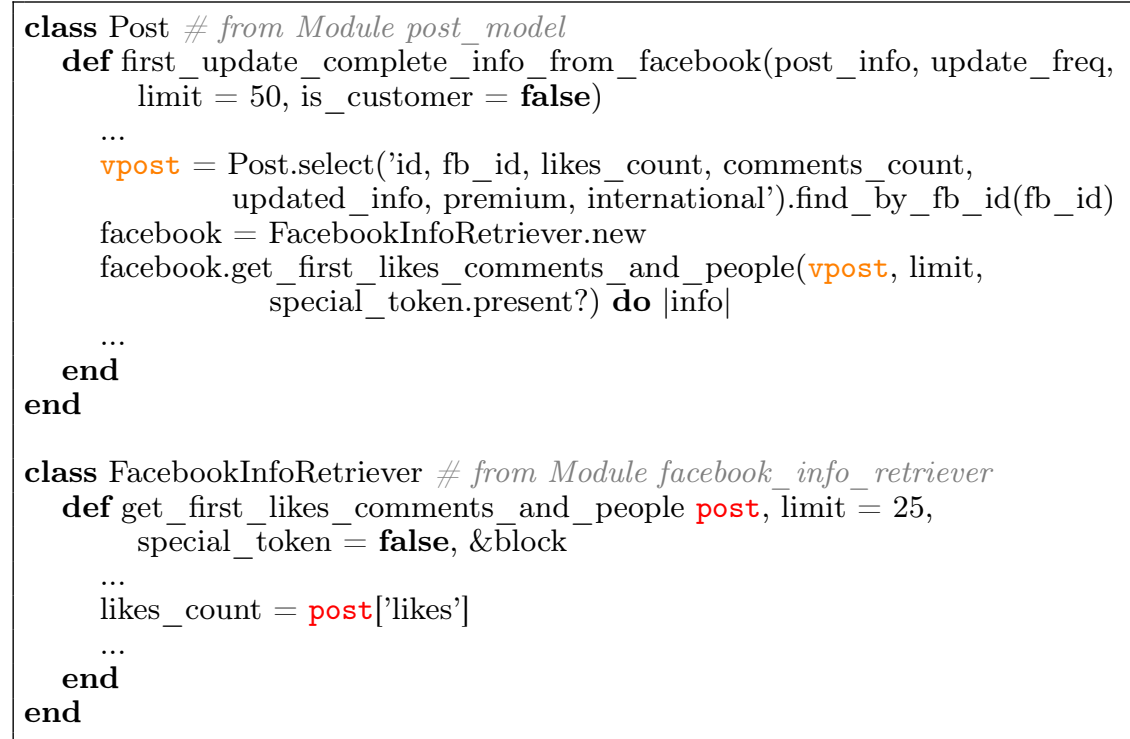

Figure 11 - Example \#2 - Divergence detected in Dito Social by type propagation

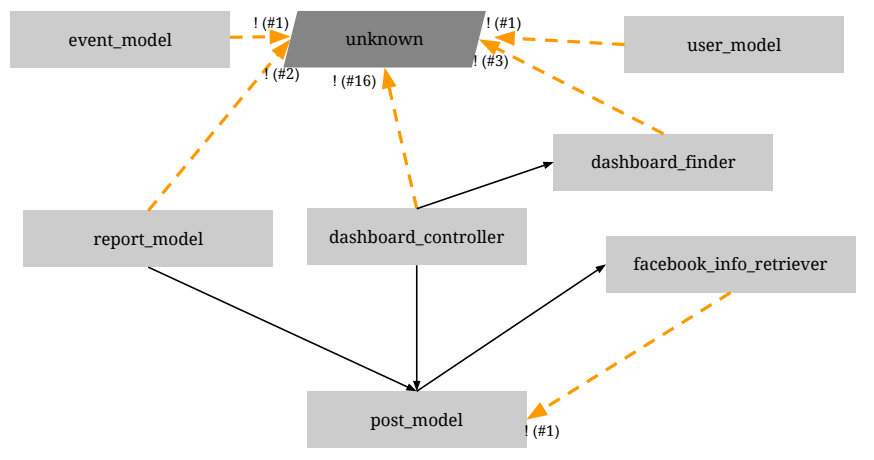

Figure 12 - Fragment of the reflexion model of Dito Social

\subsubsection{Discussion}

The software architect described the architectural rules incrementally. According to the architect, this facilitates the refinement of some rules to avoid false positives in the architecture conformance checking process. It is worth noting that the architect relied on the textual violation report to remember details about the old parts of the system and to refine the architectural rules.

Although the feature responsible for sending e-mail had already been removed from the user interface, it still is in the source code. The architect reported that unused code impacts negatively on the maintainability because it may mislead new developers. Moreover, another critical divergence was found between modules facebook_info_retriever and post_model. Module facebook_info_retriever 
is likely to be used with only the Facebook API, i.e., it cannot rely on other parts of the system. According to the architect, this divergence hampers the evolution of the system since module facebook_info_retriever is coupled with other parts. Last, the architect argued that, as the number of modules grows, the reflexion model becomes hard to analyze. Particularly in this case study, we also presented the DSM of the system. The architect argue that the two models are complementar, e.g., DSMs are much more appropriate for tasks that require a complete view of the system, but reflexion models are more appropriate to analyze dependencies among few modules.

\subsection{Tim Beta}

Architectural rules specification: The software architect specified 43 modules and seven architectural rules. A relevant subset of the specification is reported in Figure 13. Module models implements the Model layer of the MVC architectural pattern and hence can access the modules that are responsible for the data persistence (lines 3-7). Module core implements the main features of the system and hence must access the modules that provide underlying services (lines 11-14). Module workers is responsible for background activities, e.g., updating users' information based on their facebook profile afterwards they sign in (line 18).

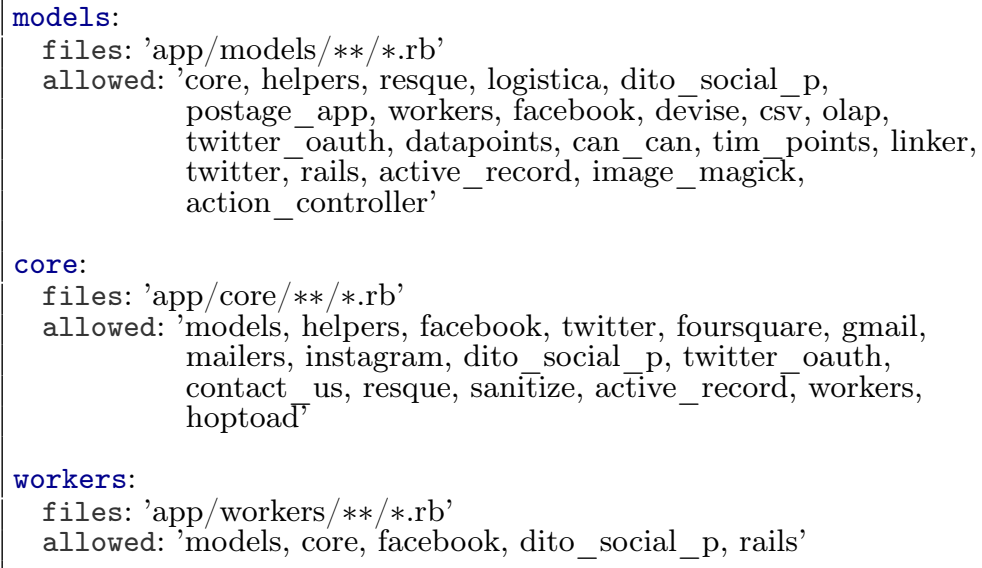

Figure 13 - Subset of the architectural specification of Tim Beta

Architectural conformance: ArchRuby could detect 22 violations in Tim Beta, as reported in Table 3. An example of a detected violation is discussed next.

Table 3 - Architectural violations detected in Tim Beta

\begin{tabular}{lllc}
\hline Module & Rules & & \# Violations \\
\hline core & allowed: 'models,$\ldots$, & 6 \\
models & allowed: & 'core,$\ldots$, & 16 \\
\hline
\end{tabular}

Example of violation \#3: Features related to the Orkut social network have been removed from Tim Beta; consequently, the respective source code has been removed as well. Nevertheless, as shown is Figure 14, class User-which belongs to module models - accesses class Core::Datapoints::Orkut (line 2), which is not explicitly 
allowed according to the architectural rules (lines 3-7 of Figure 13).

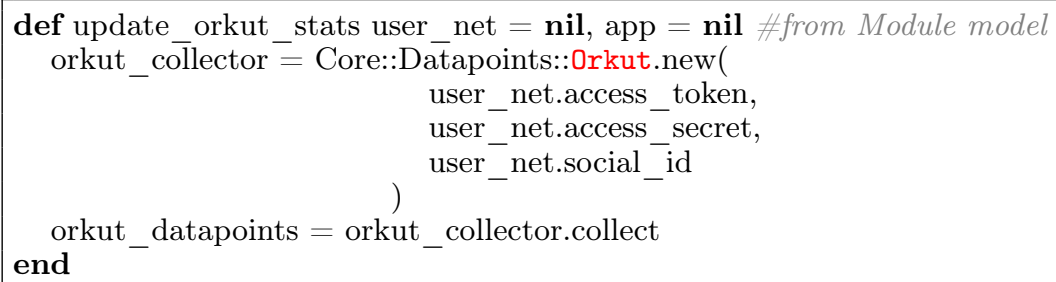

Figure 14 - Example \#3 - Divergence detected in Tim Beta

Architectural visualization: Figure 15 illustrates a fragment of the reflexion model to better visualize some identified violations. We can note divergences (orange edges) from modules core and models to classes that do not belong to any defined module; the latter refers to the scenario described in Example \#3.

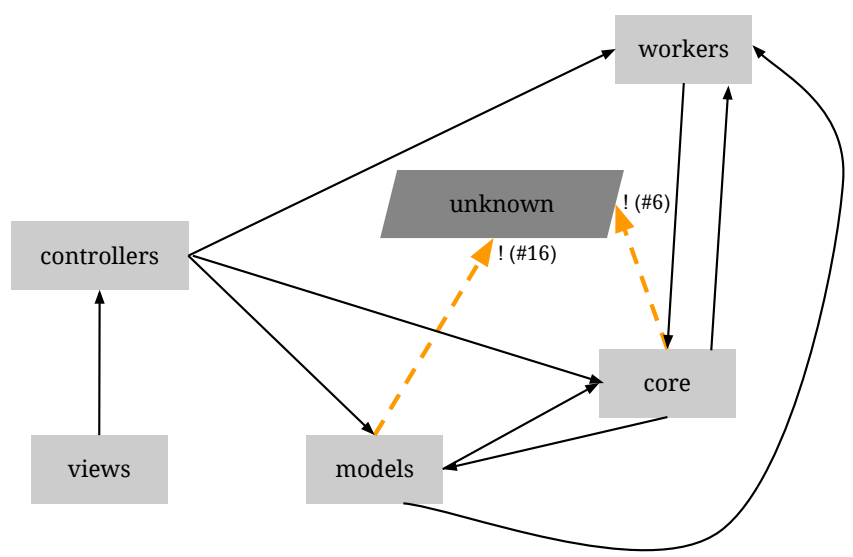

Figure 15 - Fragment of the reflexion model of Tim Beta

\subsubsection{Discussion}

The architect of Tim Beta relied on an artifact that specifies the most important modules in the system as the basis to specify the architectural rules. As a consequence, there is a relative small number of architectural rules. The architectural conformance process detected components that the software architect had thought no longer exists. For instance, all functionality related to the Orkut social network should have been entirely removed from the source code, but they were still found in the source code. Moreover, the architect argue (i) that ArchRuby is important to support the architectural monitoring since it is impractical to manually do this process; (ii) that ArchRuby should be incorporated into the continuous integration process; and (iii) that the reflexion model can be used by new team developers to understand the system modularization. 


\subsection{PLC Attorneys}

Architectural rules specification: The software architect specified 14 modules and 11 architectural rules. A relevant subset of the specification is presented in Figure 16. The purpose of the system is to keep the customer aware of the tasks that have been resolved and the ones that are still pendent. Therefore, module project is responsible for handling data about the customers' project and can access modules that contain data it needs (line 4). Module project relations is responsible for storing customer data, reporting progress, and displaying charts, and hence can access modules that provide underlying services (line 12). Finally, module mailers is responsible for triggering e-mails to clients and can access modules that provide information about the projects (line 17).

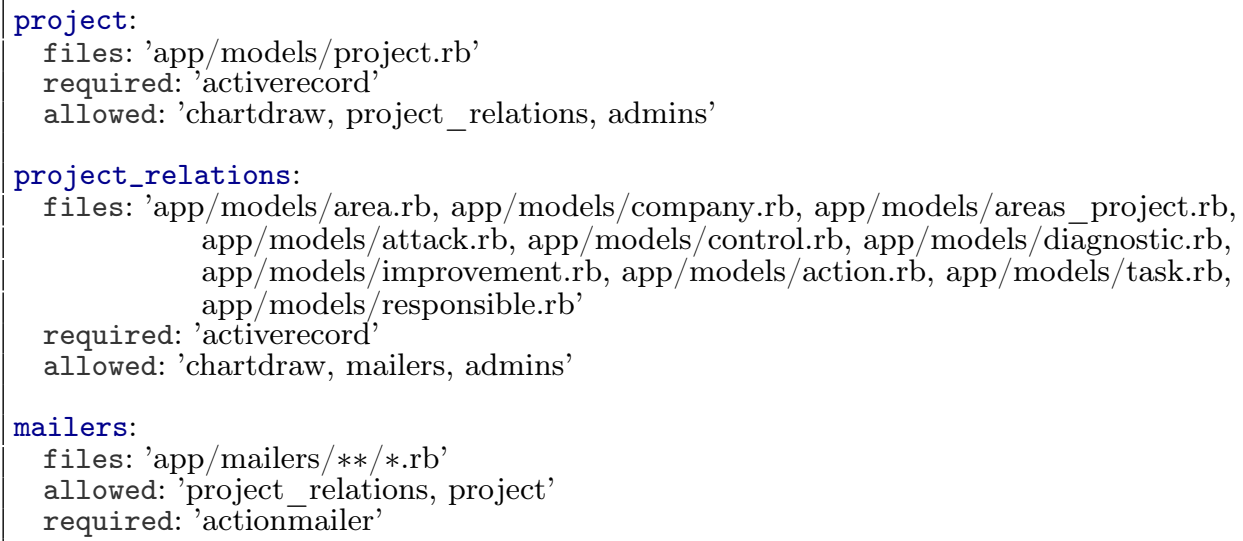

Figure 16 - Subset of the architectural specification of PLC Attorneys

Architectural conformance: ArchRuby could detect two violations in PLC Attorneys, as reported in Table 4. We argue that the small number of violations is because the system is small and it is in the beginning of the development, which contributes to developers to commit fewer architectural mistakes. However, we found a critical violation that must be corrected before deploying the system to the production environment. This violation is discussed next.

Table 4 - Architectural violations detected in PLC Attorneys

\begin{tabular}{lllc}
\hline Module & Rules & \# Violations \\
\hline mailers & required: & 'actionmailer' & 1 \\
controller & allowed: & 'presenters, ...' & 1 \\
\hline
\end{tabular}

Example of violation \#4: The e-mail delivery service relies on Gem ActionMailer for the task of sending e-mails. Nevertheless, as shown is Figure 17, class DiagnosticsMailer - which belongs to module mailers-does not establish dependency with the aforementioned Gem, which is required according to the architectural rules (line 17 of Figure 16). This violation is inevitably critical because the e-mails will not be delivered without the establishment of the dependency with ActionMailer. 


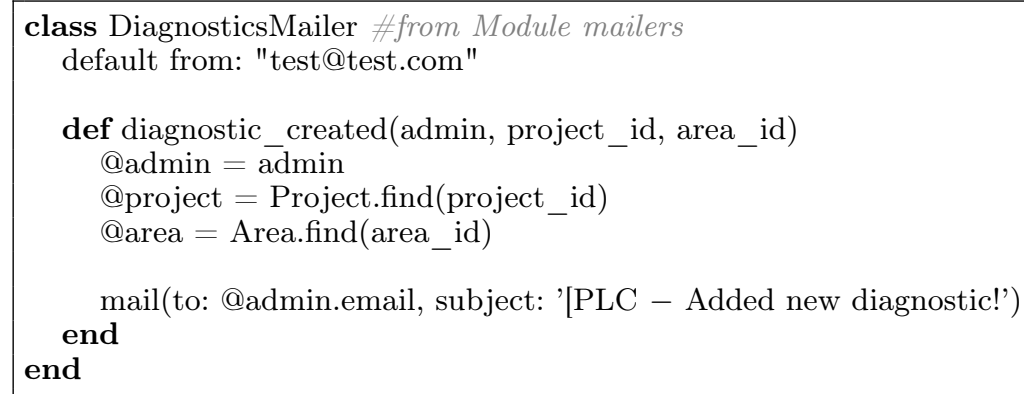

Figure 17 - Example \#4 - Absence detected in PLC Attorneys

Architectural visualization: Figure 18 illustrates a fragment of the reflexion model to better visualize some identified violations. We note an absence (red edge) from module mailers to module actionmailer, which refers to the scenario described in Example \#4. We also note a divergence (orange edge) from module controller to documents.

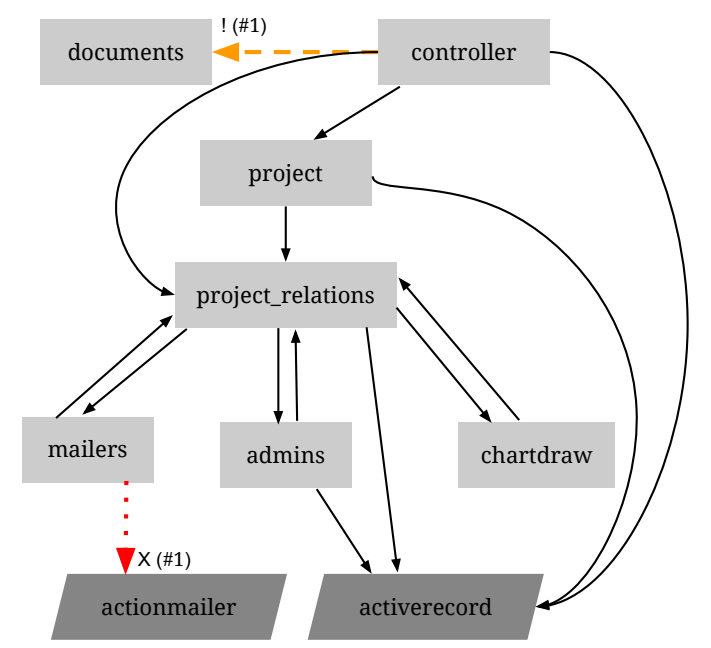

Figure 18 - Fragment of the reflexion model of PLC

\subsubsection{Discussion}

Since the system is in the early stages of its development, the number of architectural rules is relatively small. This also leads to a small number of architectural violations and thereafter to a small number of violations detected by ArchRuby. Nevertheless, ArchRuby could detect a serious architectural violation. The developer have not established a required dependency between modules mailers and actionmailer. Without such dependency, the system was unable to send e-mails. The architect reported this violation as a serious one because it breaks a core feature of the system. The architect also suggests that ArchRuby should provide means to automatically specify the architectural rules in order to minimize the effort by the software architect. 


\subsection{General Discussion}

It is important to highlight some points about the evaluation described in this section: (i) the software architects occasionally had to refine the architectural rules in order to avoid false positives after the architectural conformance process, which indicates thatin practice - the architectural rules specification and architectural conformance steps are jointly done; (ii) we could detect a high number of divergences in Dito Social and Tim Beta, which shows that developers establish dependencies with modules that are forbidden (or not explicitly allowed) by the architectural rules; (iii) on the other hand, we could detected few violations in PLC Attorneys. Since the system is new and small, we argue that these properties contribute to developers to commit fewer architectural mistakes; (iv) the software architects had no previous knowledge on the identified violations and reported that they negatively impact on the maintenance of the systems; (v) since we rely primarily on reflexion models, the software architects reported issues on visualizing the architectural violations as the number of modules grows, suggesting a scalability problem. In such cases, we allowed the architect to switch the high-level architectural model to DSM; and (vi) the software architects claimed the need for tool support to automatically monitor the source code and perform the architecture conformance checking process.

\subsection{Threats to Validity}

There are two main threats to validity of the study $\left[\mathrm{WRH}^{+} 12\right]$. First, as usual in empirical studies in software engineering, we cannot claim that our approach will provide equivalent results in other systems (external validity). However, we rely on three real-world systems that have being developed by different teams. Second, we relied on three software architects (one per system) to define the rules, to validate the detected violations, and to analyze the visualization model. As typical in human-based classifications, our results might be affected by some degree of subjectivity (construct validity). However, it is important to highlight that we interviewed the software architects who designed the evaluated architectures, and are responsible for their maintenance and evolution. Therefore, they are the right experts to evaluate our proposed approach.

\section{Effectiveness of the Type Propagation Heuristic}

Our type propagation mechanism, as described in Section 4, aims to raise the effectiveness of our approach by increasing the number of analyzed dependencies. In this section - based on the data of our previous evaluation - we provide a quantitative and qualitative discussion on the number and importance of the types inferred by our heuristic (effectiveness).

We observed, in the previous evaluation, that some architectural violations are only detected due to our heuristic. Therefore, we investigated in the three previous evaluated real-world systems - Dito Social, Tim Beta, and PLC Attorneys - the number of types and violations that are only inferred and detected, respectively, due to our heuristic.

\subsection{Research Questions}

We conducted a study to address the following overarching research questions: 
$\boldsymbol{R} \boldsymbol{Q} \# \mathbf{1}$ - How many types are only inferred due to our heuristic?

$\boldsymbol{R} \boldsymbol{Q} \# \mathbf{Q}$ - How many violations are only detected due to our heuristic?

\subsection{Data Set}

Our study mainly relies on the three previously evaluated real-world systems-namely Dito Social, Tim Beta, and PLC Attorneys-, w.r.t. the number of types and violations that are only inferred and detected, respectively, due to our heuristic.

\subsection{Results and Discussion}

In this section, we provide answers for the proposed research questions.

\subsubsection{RQ \#1: How many types are only inferred due to our heuristic?}

Our approach relies on static code analysis techniques to extract the dependencies that should be verified according to the planned architecture. Our heuristic ensures the propagation of the inferred types. Otherwise, only direct inferences of types (e.g., instantiation) would be considered.

Methodology: In order to quantify the number of types that are exclusively inferred by our heuristic, we performed ArchRuby in the three systems, enabling and disabling our type propagation heuristic. It is important to differentiate (i) the number of language features, which refers to expressions, statements, and declarations; (ii) the number of dependencies, which refers to every single dependency inspected by the conformance process; and (iii) the number of inferred types, which refers to every single triple [method, var_name, type] in set TYPES, as previously explained in Section 4. Thereupon, the number of language features is far higher than the number of dependencies, which, in turn, is far higher than the number of inferred types. For instance, assume the piece of code in Figure 19. There are seven language features, two dependencies to be inspected by the conformance process (a instantiation of and a method call from class Test to type $\mathrm{Z}$ ), and only one inferred type ([Test::bar, $\mathrm{x}, \mathrm{Z}]$ ).

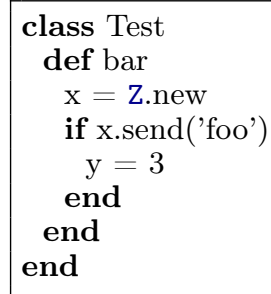

Figure 19 - Number of language features, dependencies, and inferred types

Results and Discussion: Table 5 reports our results. On the average, the percentage of additional types is $4.59 \%$. In fact, PLC Attorneys was the only system that presented percentagem below $5 \%$ since it represents a relative small system in the early stages of its development. 
Table 5 - Number of inferred types by our proposed type propagation heuristic

\begin{tabular}{lccc}
\hline Project & $\begin{array}{c}\text { \# of inferred types } \\
\text { without heuristic }\end{array}$ & $\begin{array}{c}\text { \# of inferred types } \\
\text { with heuristic }\end{array}$ & \% added \\
\hline Dito Social & 566 & 598 & $5.65 \%$ \\
Tim Beta & 672 & 709 & $5.51 \%$ \\
PLC Attorneys & 154 & 158 & $2.60 \%$ \\
\hline
\end{tabular}

In order to provide an answer in a broader context, we complementarily replicated our experiment in a data set of 28 open-source Ruby, as described in Appendix B. As reported in Table 6, the percentage of additional types is $5.03 \% \pm 3.95 \%$ (average \pm standard deviation). Statistically, the number of additional types should fall between $3.50 \%$ and $6.56 \%$ within a $95 \%$ confidence interval.

Table 6 - Complementary analysis on the number of inferred types by our heuristic

\begin{tabular}{|c|c|c|c|}
\hline Project & $\begin{array}{l}\text { \# of inferred types } \\
\text { without heuristic }\end{array}$ & $\begin{array}{l}\text { \# of inferred types } \\
\text { with heuristic }\end{array}$ & $\%$ added \\
\hline Active Admin & 345 & 349 & $1.16 \%$ \\
\hline CanCan & 26 & 26 & $0.00 \%$ \\
\hline Capistrano & 39 & 39 & $0.00 \%$ \\
\hline Capybara & 155 & 166 & $7.10 \%$ \\
\hline CarrierWave & 81 & 85 & $4.94 \%$ \\
\hline CocoaPods & 438 & 465 & $6.16 \%$ \\
\hline DevDocs & 283 & 292 & $3.18 \%$ \\
\hline Devise & 114 & 121 & $6.14 \%$ \\
\hline diaspora* & 934 & 952 & $1.93 \%$ \\
\hline Discourse & 2,950 & 3,124 & $5.90 \%$ \\
\hline FPM & 157 & 172 & $9.55 \%$ \\
\hline GitLab & 1,750 & 1,794 & $2.51 \%$ \\
\hline Grape & 137 & 146 & $6.57 \%$ \\
\hline Homebrew-Cask & 426 & 443 & $3.99 \%$ \\
\hline Homebrew & 8,026 & 8,125 & $1.23 \%$ \\
\hline Huginn & 463 & 477 & $3.02 \%$ \\
\hline Jekyll & 259 & 273 & $5.41 \%$ \\
\hline Octopress & 95 & 111 & $16.84 \%$ \\
\hline Paperclip & 132 & 137 & $3.79 \%$ \\
\hline Rails & 2,464 & 2,559 & $3.86 \%$ \\
\hline RailsAdmin & 231 & 234 & $1.30 \%$ \\
\hline Resque & 62 & 68 & $9.68 \%$ \\
\hline Ruby & 4,116 & 4,391 & $6.68 \%$ \\
\hline Sass & 519 & 560 & $7.90 \%$ \\
\hline Simple Form & 113 & 115 & $1.77 \%$ \\
\hline Spree & 1,311 & 1,324 & $0.99 \%$ \\
\hline Vagrant & 586 & 620 & $5.80 \%$ \\
\hline \multirow[t]{3}{*}{ Whenever } & 15 & 17 & $13.33 \%$ \\
\hline & & Average & $5.03 \%$ \\
\hline & & Std Dev & $3.95 \%$ \\
\hline
\end{tabular}

After a qualitative analysis of our results, although it is not trivial, we observed that our heuristic could infer more types if it also analyses and propagates the return type of method invocations. For instance, assume the piece of code in Figure 20. In this example, set TYPES would contain the tuples [Clazz::foo,y, A] and 
[Clazz::bar, z, B], but if it could infer [Clazz::foo, x, B] by analyzing the returned type, it would also include $[\mathrm{A}:: q u x, \mathrm{k}, \mathrm{B}]$ (where $k$ is the name of the formal parameter in $A:$ :qux) in the set, which promotes the type propagation through the system.

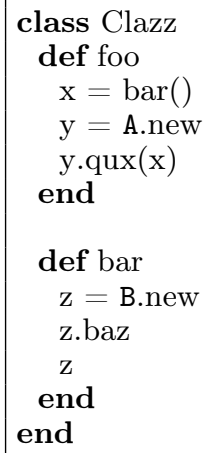

Figure 20 - Potential improvement to the type propagation heuristic

Final Remarks: Our heuristic increased the number of inspected dependencies by $5 \%$ on the average, but could increase up to $17 \%$. We argue that the increase depends on the underlying programming style. For example, our heuristic achieves better results when developers largely rely on the dependency injection design pattern [Met12].

\subsubsection{RQ \#2: How many violations are only detected due to our heuristic?}

The previous research question showed that our heuristic may increase the number of inspected types in $5 \%$ on the average. Nonetheless, it is important to investigate whether these additional types contribute to the detection of architectural violations in real scenarios.

Methodology: In order to measure the effectivity of our heuristic, i.e., the number of violations that are identified exclusively by our heuristic, we re-performed ArchRuby in the three real-world systems previously evaluated in Section 6.1, enabling and disabling the type propagation heuristic.

Results and Discussion: From the 48 architectural violations detected in the three real-world systems, three violations are detected exclusively by our heuristic. Therefore, a preliminary analysis may point out the ineffectiveness of our heuristic. However, we claim that our heuristic was indeed very effective. For example, we found 24 violations on Dito Social. On one hand, from the 566 inferred types without our heuristic, we found 22 violations $(3.89 \%)$; on the other hand, from the 32 inferred types by our heuristic, we could find two more violations (6.25\%). Likewise, we found 22 violations on Tim Beta. On one hand, from the 672 inferred types without the heuristic, we found 21 violations $(3.13 \%)$; on the other hand, from the 37 inferred types by our heuristic, we could find one more violation $(2.70 \%)$.

Figure 21 illustrates one of the violations that could be detected exclusively by our heuristic. There are forbidden accesses to class Core::Datapoints::0rkut (lines 7, 9, and 16), which are forbidden since features related to the Orkut social network have been removed from Tim Beta. Specifically for the latest violation (line 16), our approach could only detect it due to our heuristic, since the type 
was first inferred in method verify orkut users friends (line 7), but it was propagated to the formal parameter collector (line $\overline{15}$ ) through the method call to check_friends_count (line 9).

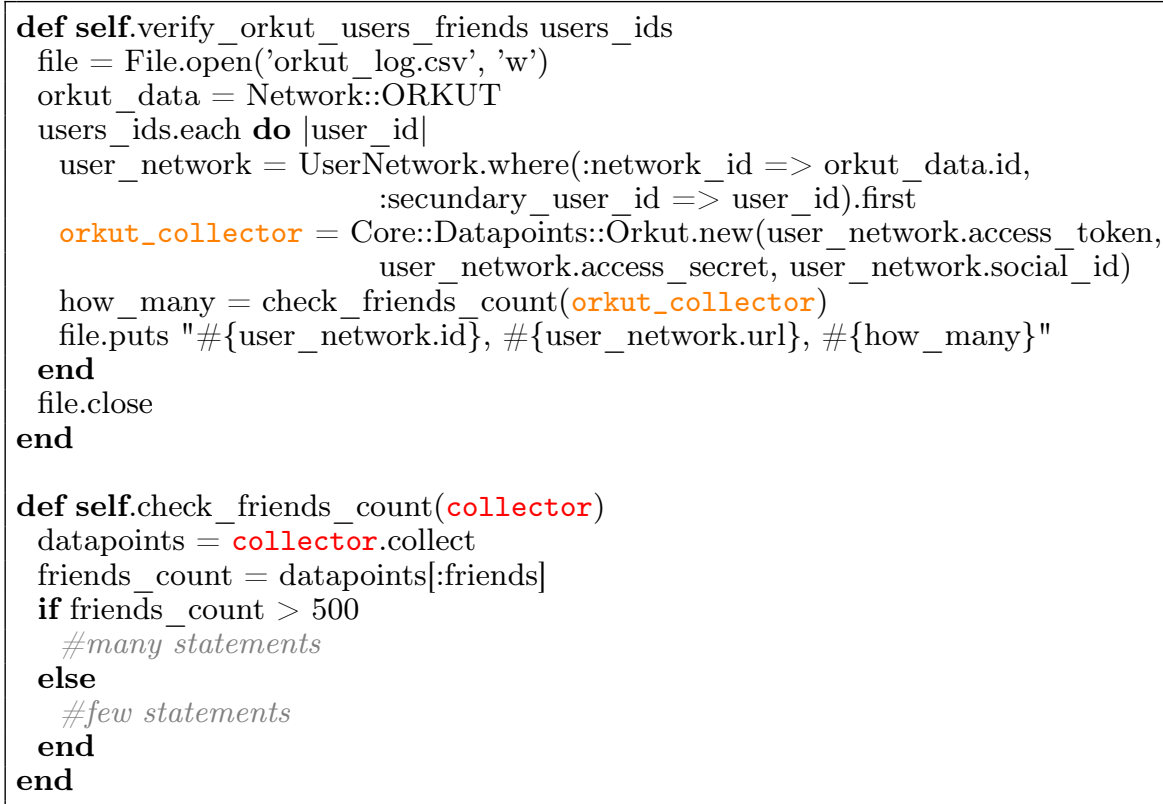

Figure 21 - Divergence detected in Tim Beta using the type propagation heuristic

Final Remarks: Some violations are identified exclusively by our heuristic. Disregarding the PLC Attorneys system where there are no violations detected by the proposed heuristic, the overall percentage of the violations identified exclusively by our heuristic is $4.35 \%(3 / 69)$, while in the remainder of the system is $3.47 \%(43 / 1,238)$.

\section{Related Work}

We organize related work in three sections: Architecture Conformance Techniques and Tools (Section 8.1); (b) Studies using Dynamic Languages (Section 8.2); and (c) Ruby Tools (Section 8.3).

\subsection{Architecture Conformance Techniques and Tools}

Our solution is inspired by DCLsuite, an architectural conformance and repair approach for Java systems [TV09, TVCB15, TV08]. Software architects define a set of constraints using the DCL language, and the tool detects architectural violations and provides suggestions on how to solve them. By contrast, ArchRuby targets a dynamic language (which required a definition and implementation of a type propagation heuristic), allows the specification of architectural constraints in YAML files, and provides two high-level architectural models to better visualize the identified violations; however, ArchRuby does not contemplate architectural repair. 
Several domain-specific languages have been proposed for architecture conformance. SCL (Structural Constraint Language) [HH06] is a Prolog-like DSL to specify and check design intent in $\mathrm{C}++$ or Java code. LogEn [EKKM08] is another Prolog-like language for defining and continuous checking structural dependencies in Java systems. The language has a explicit type predicate, where the first argument is a source element and the second parameter is its type name. DesignWizard [BGF11] is an internal DSL for detecting design and architectural anomalies in Java-based software architectures. Developers express the desired architecture by writing tests that make assertions about the structure of the Java code. Therefore, these DSLs are proposed for static languages, typically Java and $\mathrm{C}++$, and heavily depend on type information Soul [MKPW06] is a Prolog-like language that provides access to the static structure of Smalltalk programs (a dynamic language, like Ruby). However, Soul does not aim to detect architectural violations (divergences and absences), but to enforce source code regularities (e.g., all visitor methods must start with visit and must be implemented in a method protocol called action).

The high-level architectural model provided by ArchRuby is inspired by SAVE [KMNL06], an approach based on reflection models [MNS95]. SAVE compares (i) the planned architecture, as idealized by the software architect, to (ii) the implemented architecture, as extracted from source code. As a result, the tool computes the reflection model that highlights divergences and absences between these two models. Reflexion models, however, become unreadable as the number of modules and dependencies grows. Thereupon, the other high-level architectural model provided by ArchRuby is inspired by Lattix LDM, an approach based on DSMs [SJSJ05], which relies on matrices that scale better than graphs. The high-level architectural models produced by ArchRuby is very similar to a reflexion model and a DSM, although it is generated from textual architectural rules. More important, developers using ArchRuby can decide for the more appropriate high-level model to be used.

ArchLint $\left[\mathrm{MVA}^{+} 13, \mathrm{MVT}^{+} 16\right]$ proposes a novel approach to detect architectural violations, by mining version repositories. However, the systems relies on heuristics to detect patterns of dependencies between modules, which are centered on type information. Inspired on the Z language, LePUS [Ede01] is a formal language for specifying object-oriented design and architectural patterns. However, instead of checking architecture intent, LePUS focuses on the specification of programming protocols, such as forward (that holds when the formal arguments of a method are used to call a method with the same signature) and produce (a special kind of create in which the new object is used in a return statement). The language also includes a visual version, called LePUS3 [GNE08].

\subsection{Studies using Dynamic Languages}

Richards et al. [RHBV11] evaluated the use of eval in JavaScript, based on a corpus of more than 10,000 popular web sites. Unlike our findings for Ruby, they report that eval is popular and do not necessarily harmful. It is usually considered a best practice for specific tasks, such as loading scripts or data asynchronously. The authors also investigated a broad range of JavaScript dynamic features [RLBV10]. They concluded for example that libraries often change the prototype links dynamically, but such changes are restricted to built-in types, such as Object and Array, and changes in user-created types are more rare.

Hills et al. [HKV13] report a study over a significant corpus of open-source PHP systems to understand how developers actually use dynamic features, including dynamic 
file inclusion, handlers for unimplemented methods or fields, an eval expression for executing arbitrary PHP code at runtime, and variadic functions. The authors conclude that a large number of uses of dynamic features could be replaced by static ones without changing the expected behavior. Another group of researchers reached the same conclusion in a case study over a 1,000 systems developed using the Pharo language [CRTR13]. Orru et al. [OTMT15] analyzed a collection of 51 software systems written in Python, to shed light on how they use inheritance. They show that fewer classes inherit from other classes (w.r.t. Java), but more classes are inherited from. They also claim that the dataset used in this study can help to foster further research on Python software $\left[\mathrm{OTM}^{+} 15\right]$.

Hanenberb et al. [Han10] show that dynamic languages can produce high-quality systems, but requiring less time than when using a static language. They report a laboratory study when groups of developers implemented a lexical analyzer in two versions of a language, with static and dynamic types, respectively. The implementation using a static language required 161 hours, while the implementation using the dynamic version of the language required 108 hours.

\subsection{Ruby Tools}

In the Ruby ecosystem, we are not aware of any architectural conformance and visualization technique as the one proposed in this paper. However, there are several tools that aim to increase the quality of Ruby systems through static code analysis techniques. Code $\mathrm{Climate}^{10}$ is a tool that assists in code reviews. It reports where the system has to improve, e.g., complex methods, security breaches, refactoring opportunities, etc. The tool also suggests reference literature for developers to better understand the problem and the correction actions. Rubocop ${ }^{11}$ is a Gem that performs static code analysis to verify error and style rules, likewise LASER ${ }^{12}$ and ruby-lint ${ }^{13}$. In another line, Brakeman ${ }^{14}$ is a security vulnerability scanner and Pelusa ${ }^{15}$ indicates possible red flags or missing best practices. ArchRuby, in turn, complements these tools by providing means to control the architectural erosion process.

\section{Conclusion}

Software architecture erosion is a recurrent problem in software development. Deviations from the planned architecture have strong impact on the system maintainability and evolvability, and may even lead to the rewriting of components. Even more critical, the erosion process might be even more severe in dynamic languages because (i) the dynamic constructs provided by such languages make developers more propitious to break the architecture, and (ii) the developers of dynamic languages lack tool support for monitoring the implemented architecture.

To address these shortcomings, this paper described an architectural conformance and visualization approach based on static code analysis techniques and a lightweight type propagation heuristic. Such a solution provides means to control the architectural erosion process by reporting architectural violations and visualizing them in two

\footnotetext{
${ }^{10}$ https : // codeclimate.com/

${ }^{11}$ https://github.com/bbatsov/rubocop

12 https : //github.com/michaeledgar/laser

13 https : //github.com/YorickPeterse/ruby - lint

${ }^{14}$ http : //brakemanscanner.org/

${ }^{15}$ https : //github.com/codegram/pelusa
} 
high-level architectural models, namely reflexion models and DSMs. This paper also presented a tool - called ArchRuby - that implements our approach.

We evaluated our solution in three real-world systems identifying 48 architectural violations of which the developers had no prior knowledge. We also evaluated our type propagation heuristic in 28 open-source systems, reporting that (i) the number of analyzed types raises $5 \%$ on the average, but it may increase up to $17 \%$; and (ii) certain violations are only detected due to our heuristic. As a practical result, ArchRuby was integrated into the software development process adopted by Dito, the company responsible for the evaluated systems.

Ideas for future work include (i) incorporating an architectural repair solution that provides suggestions on how to solve the detected violations; (ii) improving our type propagation heuristic; (iii) integrating the proposed approach to mainstreams IDEs (e.g., RubyMine) for a better usability; and (iv) extending our solution to other dynamic languages.

The ArchRuby tool and its source code is public available at: http : //aserg.labsoft.dcc.ufmg.br/archruby

\section{References}

[BC99] Carliss Y. Baldwin and Kim B. Clark. Design Rules: The Power of Modularity. MIT Press, 1999.

[BGF11] Joao Brunet, Dalton Guerreiro, and Jorge Figueiredo. Structural conformance checking with design tests: An evaluation of usability and scalability. In 27th International Conference on Software Maintenance (ICSM), pages 143-152, 2011. doi:10.1109/ICSM.2011.6080781.

[Bla09] David A. Black. The Well-Grounded Rubyist. Manning, 2009.

[Bor11] Jens Borchers. Invited talk: Reengineering from a practitioner's view a personal lesson's learned assessment. In 15th European Conference on Software Maintenance and Reengineering (CSMR), pages 1-2, 2011. doi:10.1109/CSMR . 2011.63.

[Bos04] Jan Bosch. Software architecture: The next step. In First European Workshop (EWSA), pages 194-199, 2004. doi:10.1007/ 978-3-540-24769-2_14.

[CRTR13] Oscar Callaú, Romain Robbes, Éric Tanter, and David Röthlisberger. How (and why) developers use the dynamic features of programming languages: the case of smalltalk. Empirical Software Engineering, 18(6):1156-1194, 2013. doi:10.1007/s10664-012-9203-2.

[Ede01] Amnon H. Eden. Formal specification of object-oriented design. In International Conference on Multidisciplinary Design in Engineering, pages 256-263, 2001. doi:N/A.

[EKKM08] Michael Eichberg, Sven Kloppenburg, Karl Klose, and Mira Mezini. Defining and continuous checking of structural program dependencies. In 30th International Conference on Software Engineering (ICSE), pages 391-400, 2008. doi:10.1145/1368088.1368142.

[FhDAFH09] Michael Furr, Jong hoon (David) An, Jeffrey S. Foster, and Michael Hicks. Static type inference for Ruby. In 24th Symposium on Applied 
Computing (SAC), pages 1859-1866, 2009. doi:10.1145/1529282. 1529700.

[GNE08] Epameinondas Gasparis, Jonathan Nicholson, and Amnon H. Eden. LePUS3: An object-oriented design description language. In 5th International Diagrammatic Representation and Inference Conference, volume 5223 of Lecture Notes in Computer Science, pages 19-21. Springer, 2008. doi:10.1007/978-3-540-87730-1_37.

[Han10] Stefan Hanenberg. An experiment about static and dynamic type systems: Doubts about the positive impact of static type systems on development time. In Conference on Object Oriented Programming Systems Languages and Applications (OOPSLA), pages 22-35, 2010. doi : 10.1145/1869459.1869462.

[HH06] Daqing Hou and H. James Hoover. Using SCL to specify and check design intent in source code. IEEE Transactions on Software Engineering, 32(6):404-423, 2006. doi:10.1109/TSE. 2006.60.

[HKV13] Mark Hills, Paul Klint, and Jurgen J. Vinju. An empirical study of PHP feature usage: a static analysis perspective. In International Symposium on Software Testing and Analysis (ISSTA), pages 325335, 2013. doi:10.1145/2483760.2483786.

[KMHM08] Jens Knodel, Dirk Muthig, Uwe Haury, and Gerald Meier. Architecture compliance checking - experiences from successful technology transfer to industry. In 12th European Conference on Software Maintenance and Reengineering (CSMR), pages 43-52, 2008. doi:10.1109/CSMR. 2008.4493299.

[KMNL06] Jens Knodel, Dirk Muthig, Matthias Naab, and Mikael Lindvall. Static evaluation of software architectures. In 10th European Conference on Software Maintenance and Reengineering (CSMR), pages 279-294, 2006. doi:10.1109/CSMR.2006.53.

[KMR08] Jens Knodel, Dirk Muthig, and Dominik Rost. Constructive architecture compliance checking - an experiment on support by live feedback. In 24th International Conference on Software Maintenance (ICSM), pages 287-296, 2008. doi:10.1109/ICSM.2008.4658077.

[Met12] Sandi Metz. Practical Object-Oriented Design in Ruby: An Agile Primer. Addison-Wesley, 2012.

[MKPW06] Kim Mens, Andy Kellens, Frédéric Pluquet, and Roel Wuyts. Coevolving code and design with intensional views: A case study. Computer Languages, Systems \&3 Structures, 32(2-3):140-156, 2006. doi:10.1016/j.cl.2005.09.002.

[MNS95] Gail Murphy, David Notkin, and Kevin Sullivan. Software reflexion models: Bridging the gap between source and high-level models. In 3rd Symposium on Foundations of Software Engineering (FSE), pages 18-28, 1995. doi:10.1145/222124.222136.

$\left[\mathrm{MVA}^{+} 13\right] \quad$ Cristiano Maffort, Marco Tulio Valente, Nicolas Anquetil, Andre Hora, and Mariza Bigonha. Heuristics for discovering architectural violations. In 20th Working Conference on Reverse Engineering (WCRE), pages 222-231, 2013. doi:10.1109/WCRE.2013.6671297. 
[MVT15a] Sergio Miranda, Marco Tulio Valente, and Ricardo Terra. ArchRuby: Conformidade e visualização arquitetural em linguagens dinâmicas. In VI Brazilian Conference on Software: Theory and Practice (CBSoft), Tools Session, pages 1-8, 2015. doi:N/A.

[MVT15b] Sergio Miranda, Marco Tulio Valente, and Ricardo Terra. Conformidade e visualização arquitetural em linguagens dinâmicas. In $X$ VIII Ibero-American Conference on Software Engineering (CIbSE), Software Engineering Technologies (SET) Track, pages 1-14, 2015. doi:N/A.

$\left[\mathrm{MVT}^{+} 16\right] \quad$ Cristiano Maffort, Marco Tulio Valente, Ricardo Terra, Mariza Bigonha, Nicolas Anquetil, and Andre Hora. Mining architectural violations from version history. Empirical Software Engineering Journal, pages 1-42, 2016. doi:10.1007/s10664-014-9348-2.

$\left[\mathrm{OTM}^{+} 15\right] \quad$ Matteo Orrú, Ewan Tempero, Michele Marchesi, Roberto Tonelli, and Giuseppe Destefanis. A curated benchmark collection of python systems for empirical studies on software engineering. In 11th International Conference on Predictive Models and Data Analytics in Software Engineering (PROMISE), pages 1-4, 2015. doi: 10.1145/2810146.2810148.

[OTMT15] Matteo Orru, Ewan Tempero, Michele Marchesi, and Roberto Tonelli. How do Python programs use inheritance? a replication study. In 22nd Asia Pacific Software Engineering Conference (APSEC), pages 19-21, 2015. doi:N/A.

[Par94] David Lorge Parnas. Software aging. In 16th International Conference on Software Engineering (ICSE), pages 279-287, 1994. doi:10.1109/ ICSE. 1994. 296790.

[PTD $\left.{ }^{+} 10\right]$ Leonardo Passos, Ricardo Terra, Renato Diniz, Marco Tulio Valente, and Nabor Mendonça. Static architecture-conformance checking: An illustrative overview. IEEE Software, 27(5):82-89, 2010. doi: 10.1109/MS. 2009.117.

[RHBV11] Gregor Richards, Christian Hammer, Brian Burg, and Jan Vitek. The eval that men do: A large-scale study of the use of eval in JavaScript applications. In 25th European Conference on Objectoriented Programming (ECOOP), pages 1-27, 2011. doi:10.1007/ 978-3-642-22655-7_4.

[RLBV10] Gregor Richards, Sylvain Lebresne, Brian Burg, and Jan Vitek. An analysis of the dynamic behavior of JavaScript programs. In 31st Conference on Programming Language Design and Implementation (PLDI), pages 1-12, 2010. doi:10.1145/1806596.1806598.

[SGCH01] Kevin J. Sullivan, William G. Griswold, Yuanfang Cai, and Ben Hallen. The structure and value of modularity in software design. In 9th International Symposium on Foundations of Software Engineering (FSE), pages 99-108, 2001. doi:10.1145/503209.503224.

[SJSJ05] Neeraj Sangal, Ev Jordan, Vineet Sinha, and Daniel Jackson. Using dependency models to manage complex software architecture. In 20th Conference on Object-Oriented Programming, Systems, Languages, 
and Applications (OOPSLA), pages 167-176, 2005. doi:10.1145/ 1094811.1094824.

[SRK ${ }^{+}$09] Santonu Sarkar, Shubha Ramachandran, G. Sathish Kumar, Madhu K. Iyengar, K. Rangarajan, and Saravanan Sivagnanam. Modularization of a large-scale business application: A case study. IEEE Software, 26:28-35, 2009. doi:10.1109/MS.2009.42.

[TV08] Ricardo Terra and Marco Tulio Valente. Towards a dependency constraint language to manage software architectures. In 2nd European Conference on Software Architecture (ECSA), pages 256-263, 2008. doi:10.1007/978-3-540-88030-1_19.

[TV09] Ricardo Terra and Marco Tulio Valente. A dependency constraint language to manage object-oriented software architectures. Software: Practice and Experience, 32(12):1073-1094, 2009. doi:10.1002/spe. 931.

[TVCB15] Ricardo Terra, Marco Tulio Valente, Krzysztof Czarnecki, and Roberto S. Bigonha. A recommendation system for repairing violations detected by static architecture conformance checking. Software: Practice and Experience, 45(3):315-342, 2015. doi:10.1002/spe.2228.

$\left[\mathrm{WRH}^{+} 12\right] \quad$ Claes Wohlin, Per Runeson, Martin Höst, Magnus C. Ohlsson, Björn Regnell, and Anders Wesslén. Experimentation in Software Engineering. Springer, 2012. doi:10.1007/978-3-642-29044-2. 


\section{A Dependency Structure Matrices}

This appendix presents the DSMs of the systems evaluated in Section 6. Figures 22, 23, and 24 illustrate the DSM of Dito Social, Tim Beta, and PLC Attorneys, respectively.

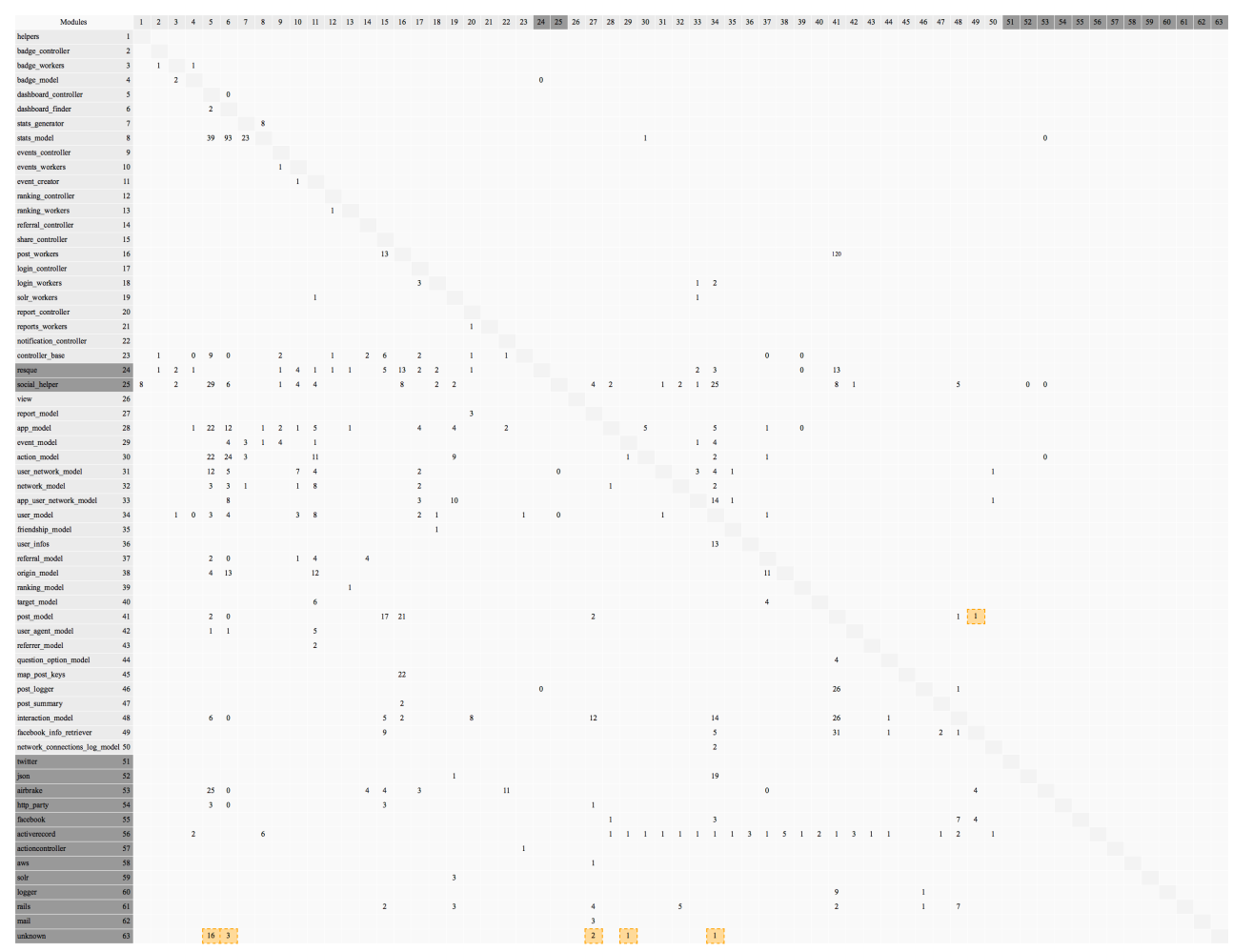

Figure 22 - DSM of Dito Social 


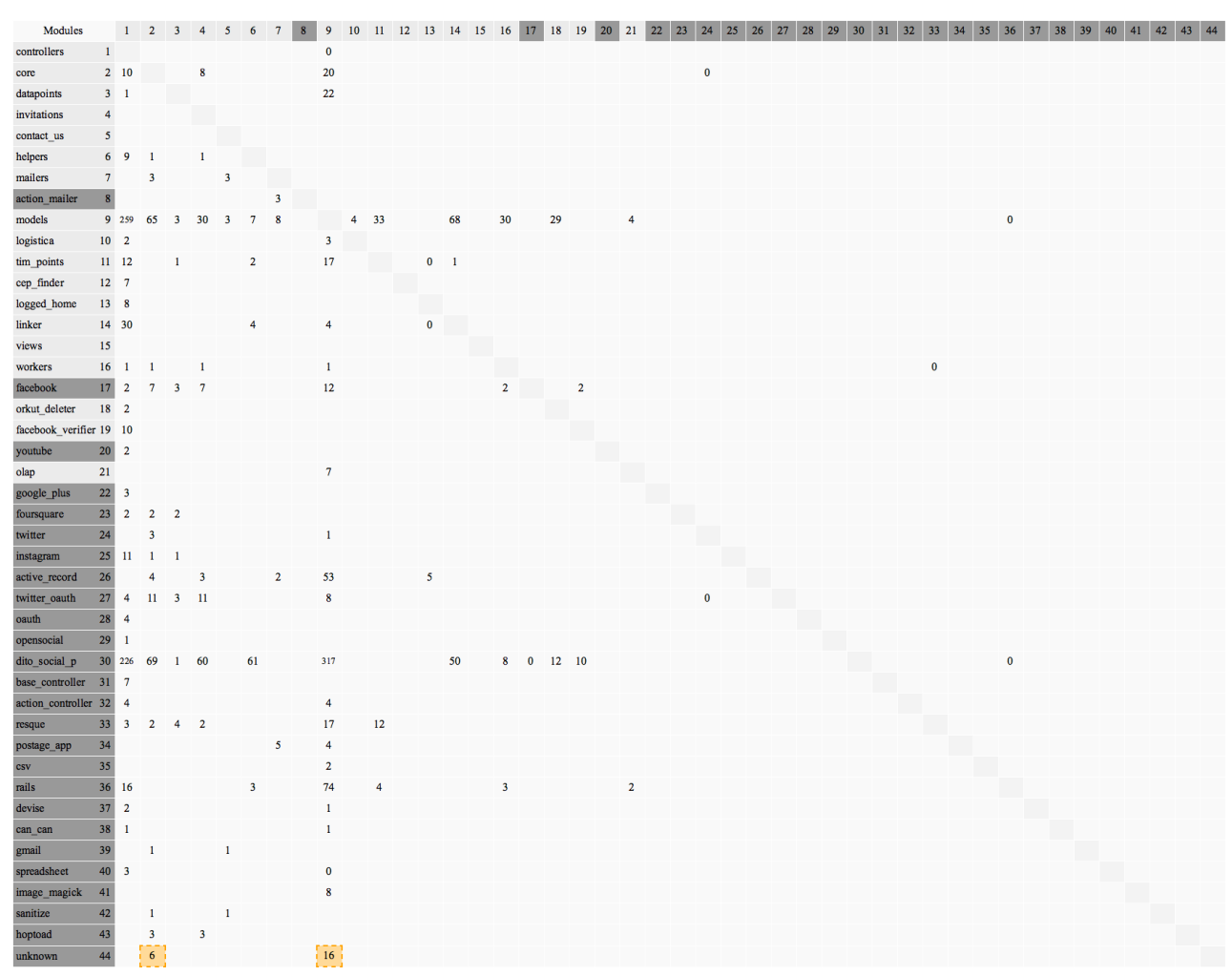

Figure 23 - DSM of Tim Beta

\begin{tabular}{|c|c|c|c|c|c|c|c|c|c|c|c|c|c|c|c|}
\hline Modules & & 1 & 2 & 3 & 4 & 5 & 6 & 7 & 8 & 9 & 10 & 11 & 12 & 13 & 14 \\
\hline controllers & 1 & & & & & & 0 & & & & & & & & \\
\hline project & 2 & 11 & & & 2 & & 1 & & & & & & & & \\
\hline project_relations & 3 & 38 & 5 & & 1 & & 1 & & & & 4 & & & & \\
\hline mailers & 4 & & & 1 & & & & & & & & & & & \\
\hline documents & 5 & 1 & & & & & & & & & & & & & \\
\hline admins & 6 & 3 & 1 & 15 & 1 & & & & & & & & & & \\
\hline consolidated_control & 17 & 9 & & & & & & & & & & & & & \\
\hline presenters & 8 & 88 & & & & & & & & & & & & & \\
\hline devise & 9 & 6 & & & & & & & & & & & & & \\
\hline chartdraw & 10 & & 5 & 5 & & & & & & & & & & & \\
\hline actioncontroller & 11 & 1 & & & & & & & & & & & & & \\
\hline activerecord & 12 & & 1 & 10 & & 4 & 3 & 2 & & & & & & & \\
\hline actionmailer & 13 & & & & 1 & & & & & & & & & & \\
\hline accesscontrol & 14 & & & & & & & & & & & & & & \\
\hline
\end{tabular}

Figure 24 - DSM of PLC Attorneys 


\section{B Open-source Data Set}

Table 7 summarizes information about our open-source data set. It contains 28 out of the 30 most starred Ruby projects in Github (on September, 2015), which represents a large and heterogeneous collection of software systems, ranging from management systems and remote server automation to frameworks and medium-sized general-purpose libraries. ${ }^{16}$ We discarded only two projects: Bootstrap for Sass, a tiny project that solely provides support to the Sass-based bootstrap CSS framework; and Software Engineering Blogs, which is not an application but a plain Ruby script that generates an $\mathrm{OPML}^{17}$ file with a list of technology web sites. In total, we analyzed over half million LOC and eight thousand rb files.

Table 7 - Evaluated open-source systems

\begin{tabular}{lrrr}
\hline Project and version & LOC & \# of rb files & \# of Gems \\
\hline Active Admin (v1.0.0.pre1) & 6,053 & 154 & 42 \\
CanCan (v1.6.10) & 878 & 16 & 13 \\
Capistrano (v3.4.0) & 2,544 & 44 & 7 \\
Capybara (v2.5.0) & 8,894 & 107 & 20 \\
CarrierWave (v0.10.0) & 2,075 & 37 & 15 \\
CocoaPods (v0.39.0.beta.4) & 8,128 & 94 & 41 \\
DevDocs (66cefbd) & 12,339 & 293 & 27 \\
Devise (v3.4.1) & 3,007 & 60 & 19 \\
diaspora* (v0.5.2.0) & 6,775 & 126 & 128 \\
Discourse (vlatestes-realease) & 14,183 & 219 & 101 \\
FPM (v1.4.0) & 3,537 & 25 & 11 \\
GitLab (v7.14.1) & 11,591 & 219 & 137 \\
Grape (v0.13.0) & 3,370 & 88 & 24 \\
Homebrew-Cask (v0.56.0) & 5,720 & 136 & 8 \\
Homebrew (8278b89) & 133,322 & 3,429 & 4 \\
Huginn (f4b8e73) & 1,464 & 18 & 90 \\
Jekyll (v3.0.0.pre.beta8) & 3,911 & 61 & 39 \\
Octopress (v2.0) & 1,313 & 23 & 13 \\
Paperclip (v4.3.0) & 3,081 & 59 & 34 \\
Rails (v4.2.4) & 55,530 & 849 & 82 \\
RailsAdmin (v0.7.0) & 4,624 & 111 & 48 \\
Resque (v1.25.0.pre) & 1,885 & 25 & 12 \\
Ruby (v2_2_3) & 170,345 & 1,076 & 0 \\
Sass (v3.4.18) & 13,080 & 130 & 8 \\
Simple Form (v3.1.0.rc2) & 2,007 & 55 & 9 \\
Spree (v3.0.4) & 5,947 & 149 & \\
Vagrant (v1.7.4) & 8,156 & 126 & 13 \\
Whenever (v0.9.4) & 632 & & \\
\hline
\end{tabular}

\footnotetext{
${ }^{16}$ https://github.com/search?l=ruby $\& p=1 \& q=$ stars $\% 3 \mathrm{~A} \% 3 \mathrm{E} 1 \& \mathrm{~s}=$ stars\&type=Repositories, as available on August 2015.

${ }^{17}$ Outline Processor Markup Language (OPLM) is an XML format for outlines, which is straightforward imported by RSS readers.
} 


\section{About the authors}

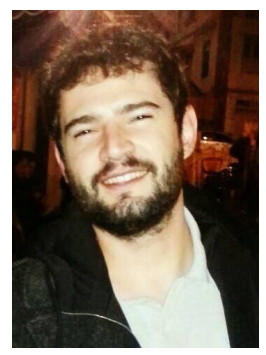

Sergio Miranda is a M.Sc. student in Computer Science at Federal University of Minas Gerais, Brazil. He has been team leader in Dito IT company for six years, working with scalability and architecture of large-scale systems. Contact him at sergio.miranda@dcc.ufmg.br.

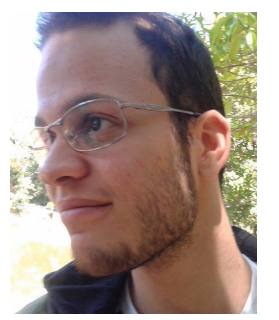

Elder Rodrigues $\mathbf{J r}$ is an undergraduate student of Computer Science at Federal University of Lavras, Brazil. He also received the technical high school degree in Informatics from CEFET-MG, Brazil (2012). He has worked as teaching assistant of Algorithms and Data Structures for one year. Currently, he holds a CNPq junior research grant to work on architectural conformance. Contact him at elderjr@computacao.ufla.br.

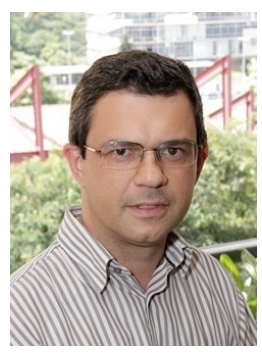

Marco Tulio Valente received his $\mathrm{PhD}$ degree in Computer Science from the Federal University of Minas Gerais, Brazil (2002), where he is an assistant professor in the Computer Science Department, since 2010. His research interests include software architecture and modularity, software maintenance and evolution, and software quality analysis. He is a "Researcher I-D" of the Brazilian National Research Council (CNPq). He also holds a "Researcher from Minas Gerais State" scholarship, from FAPEMIG. Valente has co-authored more than 80 refereed papers in international conferences and journals. Currently, he heads the Applied Software Engineering Research Group (ASERG), at DCC/UFMG. Contact him at mtov@dcc.ufmg.br, or visit www.dcc.ufmg.br/ mtov.

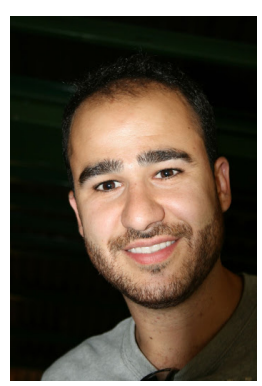

Ricardo Terra received his Ph.D. degree in Computer Science from Federal University of Minas Gerais, Brazil (2013) with a 1-year internship at the University of Waterloo, Canada. Since 2014, he is an assistant professor in the Department of Computer Science at Federal University of Lavras, Brazil. His research interests include software architecture maintainability and evolvability. Contact him at terra@dcc.ufla.br, or visit www.dcc.ufla.br/〜 terra.

Acknowledgments Our research has been supported by CAPES, FAPEMIG, and CNPq. 